\title{
Borehole Antenna Considerations in the EMRE System: Frequency Band 312.5-2500 kHz
}

\author{
Arto Korpisalo*
}

\author{
Geological Survey of Finland, P.O. Box 96, FI-02151 Espoo, Finland
}

\begin{abstract}
The ElectroMagnetic Radiofrequency Echoing (EMRE) system is utilized in radiofrequency imaging (RIM) measurements between two deep boreholes. It is based on radiowave attenuation, making it possible to reconstruct the attenuation distribution of the borehole section (tomogram). The main parts of the EMRE system are a continuous wave (CW) transmitter and superheterodyne-type receiver. The insulated dipole antennas are the transducers between the device and environment, or together they form a transmission line. The system is bistatic because the antennas are placed in separate boreholes.

When an antenna is situated in a dissipative medium, e.g. in a deep borehole, its performance becomes highly environmentally sensitive. Direct measurements of the feed-point current and radiative characteristics are impossible, and numerical models must therefore be used to investigate the borehole effects. A generalized transmission line model of an insulated antenna is a simple and useful way to examine the antenna-related properties. A drawback is that the borehole water must be excluded from the models due to the high wave number of water. The commercial simulation package FEKO is a numerical modelling package and a powerful tool when constructing more realistic antenna and borehole models. FEKO models and transmission line models (WKG, CHEN) were compared and the results coincided in the frequency band of $312.5-2500 \mathrm{kHz}$. According to the FEKO studies, the influence of borehole water and the location of the antenna in the borehole, i.e. centric or eccentric, did not appear to have a significant effect. The scattering parameter $\mathrm{s}_{11}$, the ratio of forward and returned power, is a useful antenna parameter to estimate the power level that is radiated from the antenna to the surrounding rock. According to the FEKO models, the highest measurement frequency $(2500 \mathrm{kHz})$ of the EMRE system seems to suffer the most under the borehole conditions due to the low values of $s_{11}(\sim-2 \mathrm{~dB})$. The performance level of the system determines the maximum distances (translumination distance) at which the signals are still detectable. According to the results using a simplified measurement geometry, the EMRE system can operate at distances of $<1000 \mathrm{~m}$ when the host rock has resistivities of $>10000 \Omega \mathrm{m}$ and the lowest frequency of $312.5 \mathrm{kHz}$ is used. At minor distances of 400-600 $\mathrm{m}$, the system operates well at all frequencies.
\end{abstract}

Keywords: Attenuation, EMRE, FEKO, geotomography, insulated dipole antennas, radio imaging method, RIM, transmission line model.

\section{INTRODUCTION}

The ElectroMagnetic Radiofrequency Echoing (EMRE) system operates in the frequency domain [1], and thus differs from ground penetrating $\operatorname{radar}(G P R)$ in two ways. GPR is a time domain device and usually operates in the reflection mode rather than in the transmission mode, which is familiar for the radiofrequency imaging method (RIM/EMRE). Electromagnetic methods are usually based on the fact that earth materials may have large contrasting electrical properties. When operating in the transmission mode, clearly defined boundaries are not necessities. The first steps in examining wave propagation below the Earth's surface were taken at the beginning of the 20th century. During an intensive period of development in electronics and device techniques, RIM has become a potential prospecting method, for instance for the delineation of massive sulphide deposits,

*Address correspondence to this author at the Geological Survey of Finland, P.O. Box 96, FI-02151 Espoo, Finland; Tel: +358400708140;

Fax: +358295032901; E-mail: arto.korpisalo@gtk.fi where the rock becomes gradually more conductive towards the centre of the mineralization [2-6]. Operating in the transmission mode, the system must have a separate transmitter and receiver antenna, and the system is referred to as bistatic. When the antennas are embedded in a dissipative medium, their functioning strongly depends on the electrical properties of the surrounding material. (Figs. 1, $2,3,6)$.

The physical behaviour of an electromagnetic field is mathematically governed by Maxwell equations, which describe the relationship between the electric and magnetic field in a medium and quantify the medium properties. The field depends on the distance $\bar{r}$ from the source. Certain characteristics of an electromagnetic field dominate at one particular distance from the antenna, while a completely different behaviour can dominate at another location. The wave number $\bar{k}$ multiplied by the distance $\bar{r}$ defines the behaviour [7-9]. The basic characteristics of the equations, using a plane wave solution, have been presented by Korpisalo [1]. The major factor that limits the use of 


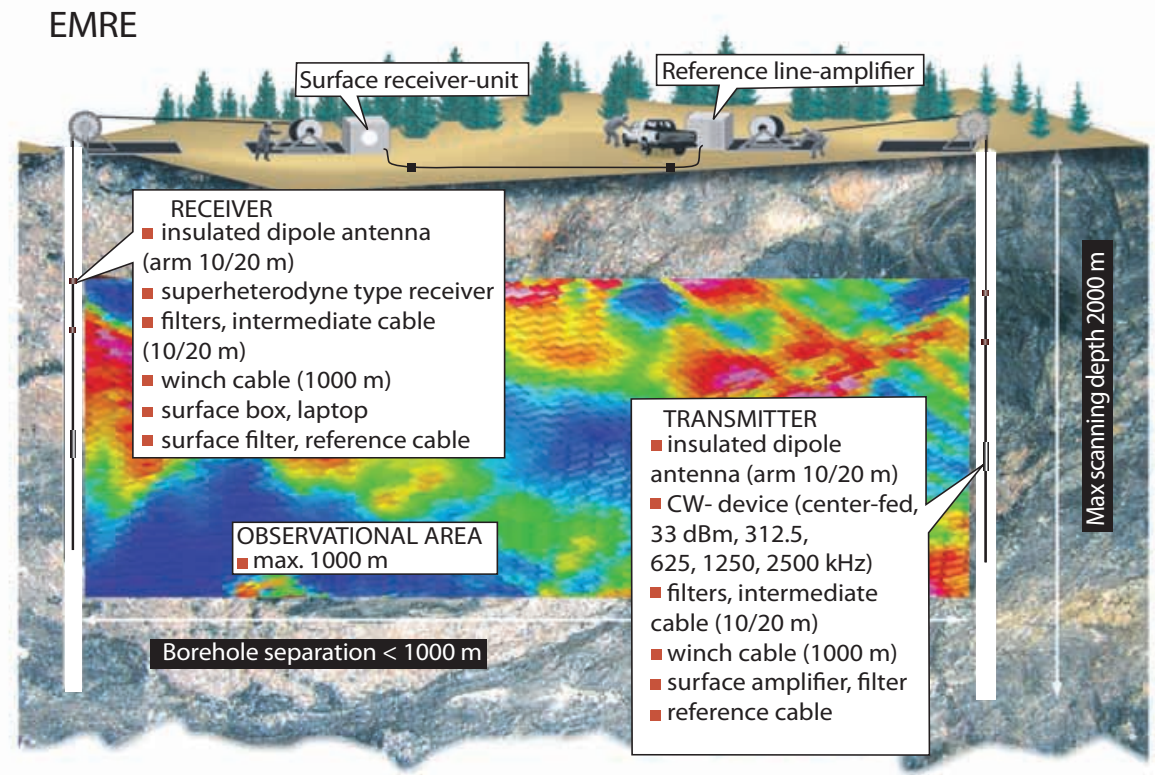

Fig. (1). The EMRE device and measurement geometry (not to scale). The mobile receiver takes measurements during movement at a frequency of $2 \mathrm{~Hz}(\Delta \sim 0.25 \mathrm{~m}, \sim 30 \mathrm{~m} / \mathrm{min})$, while the stable transmitter transmits continuously $(312.5-625-1250-2500 \mathrm{kHz})$. The attenuation distribution of the borehole section is reconstructed $(\mathrm{dB} / \mathrm{m})$ with the red colour corresponding to higher attenuation.

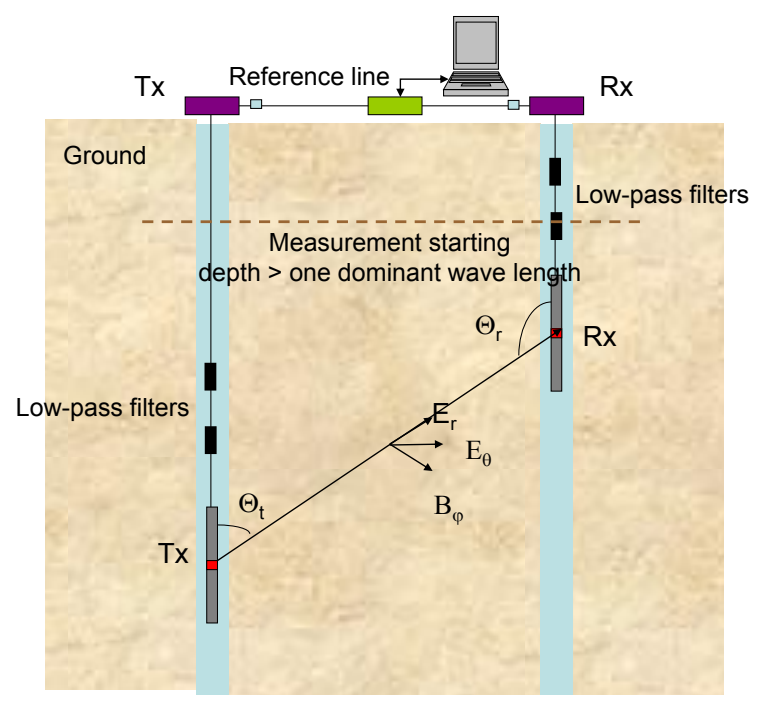

Fig. (2). Schematic drawing of the transmitter and receiver dipoles and the effective field components, showing the field components of the electric dipole. The tangential component $\mathrm{E}_{\Theta}$ is the dominant component in the far-field domain, where the radial component $E_{r}$ vanishes. The magnetic axial component $B_{\Phi}\left(=\mu H_{\Phi}\right)$ is not encountered.

radiofrequencies in geophysics is the strong absorption of energy in most earth materials, or the penetration may be highly frequency dependent. Thus, the expected attenuation rates of electromagnetic waves in a frequency band should be taken into account when estimating whether the use of the RIM method is justified in the research area [10-14].

\section{EMRE INSTRUMENT}

In Finland, the radiofrequency imaging method (RIM) has been used since the pioneering work by A. Korpisalo $[15,16]$. The heart of the system is a continuous wave $(C W)$ device that simultaneously operates at a maximum of four frequencies: $312.5,625,1250$ and $2500 \mathrm{kHz}$. The electric dipole antennas are $20 / 40 \mathrm{~m}$ long (arm lengths). The antennas are centre-fed and consist of control tubes $36 \mathrm{~mm}$ in diameter for the corresponding electronics and voltage source. The tubes are $1.5 \mathrm{~m}$ long in the transmitter and $2.5 \mathrm{~m}$ long in the receiver. Intermediate cables of $10 / 20 \mathrm{~m}$ in length are used to connect the antennas to the winch cable. Lowpass filters are used to isolate the antenna from the winch cable, the technique used in the multiband trap dipoles [17], or to cut off frequencies above the reference frequency of $156.25 \mathrm{kHz}$. The receiver and transmitter surface units are connected using a ground-level reference line where two low-pass filters are connected [1, 14].

\section{Borehole Transmitter}

Both the transmitter and receiver have internal crystals of their own. In the transmitter, the basic frequency is $2.5 \mathrm{MHz}$, which is also the highest measurement frequency. The nominal power of the transmitter is $2 \mathrm{~W}(33 \mathrm{dBm})$, but the effective output power is less. The front-end of the transmitter consists of a amplifier for each frequency. After the amplification, the discrete signals are summed to one output signal. Because the broad frequency band (312.5$2500 \mathrm{kHz}$ ) is sent simultaneously and the electrical properties of the borehole environment may be highly variable, it has not been possible to properly handle the impedance matching of the antenna, and part of the antenna power is lost due to the high voltage standing wave ratios $(V S W R)$ generated by the impedance mismatches $[17,18]$. In addition to the four measurement frequencies, the vital reference frequency $(156.25 \mathrm{kHz})$ is generated, which is fed to the earth surface through the low-pass filters. At the surface the signal is amplified and fed to the surface receiver using a groundlevel two-wire line through low-pass filters. The reference signal has an important role in the proper detection of amplitude and phase difference [14]. 


\section{Borehole Receiver}

Technically, the EMRE receiver is established in a manner that does not deviate from a normal radio. The receiver has a crystal of $2.6 \mathrm{MHz}$. The front end of the receiver ( $R F$ amplifier) and mixers are situated in the borehole unit and the normal components (e.g. detector) in the surface box. The input radiofrequency signals are first filtered and amplified before a normal mixing stage, where the four intermediate frequencies $(I F)$ are generated [18]. The IF band is broad ( four octaves) to be transferred economically through a two-wire line to the earth surface. Thus, a carrier frequency of $162.50 \mathrm{kHz}$ is used and the IF signals are modulated with the carrier signal to produce a band of one octave, which is guided more effectively to the surface receiver. The detection is carried out after the demodulation using the IF band. One important functional feature and at the same time a limiting factor in the present receiver is that its dynamic power is $\angle 40 \mathrm{~dB}$, and the user thus has to select the appropriate antennas carefully in order to avoid the saturation of the receiver. Saturation can be eliminated by changing the antennas, rejecting the problematic frequency or using longer distances between the transmitter and receiver [14].

\section{Insulated Antennas}

The EMRE antennas are symmetrical linear electric dipoles (thin cylindrical antennas) with centre-fed voltage sources. The conductive arms are surrounded by a soft rubber casing ( $16 \mathrm{~mm}$ in diameter, $\varepsilon_{r i} \approx 3.0$ ), thus effectively isolating the conductors from the environment. They are half-way antennas (open-end termination or $Z_{s}=\infty$ ) with total lengths of $20 / 40 \mathrm{~m}$, thus being physically short when compared with the corresponding wavelengths in rocks. When the length of the antenna arm is $h=\lambda / 4$, the antenna is referred to as a half-wave dipole [17]. The situation is known as a resonance and the antenna impedance is matched with the impedance of the transmitter generator. In the EMRE system it has not been possible to properly handle the impedance matching between the antenna and generator, and the high voltage standing wave ratios (VSWR) reduce the effective radiative part of the power. The current distribution determines the antenna characteristics. Electromagnetic fields do not exist in a good conductor, and the current is concentrated on the surface of the conductor, depending on its type (skin depth) [12]. Accurate calculation of the currents of an arbitrary antenna is difficult, and numerical methods must be used (e.g. FEKO/MoM) [19].

\section{BOREHOLE ANTENNA CHARACTERISTICS}

The main function of an antenna is to radiate and receive electromagnetic energy. The antennas serve as the transducers between the device and environment, or together they form a transmission line [18]. A fundamental principle of the antennas is reciprocity, meaning that the performance of an antenna is the same whether the radiation or reception is considered (same frequency and impedance). Thus, the antenna parameters could be measured either from the transmitter or receiver. The directivity $\mathrm{D}$ is the ratio of the power radiated in a particular direction to the power in the reference (isotropic) antenna. The gain $\mathrm{G}$ is a measure of how strong a signal is received or transmitted compared to an isotropic antenna. An ideal isotropic antenna radiates energy outward in a sphere with a directivity of $1(0 d B)$. The gain takes into account all the losses and can be expressed as $G=\eta D$, where $\eta$ is the antenna efficiency. The input impedance $Z_{\text {in }}$ of an antenna is an important property and is a function of the radiation resistance, resistive losses in the conductor, reactive storage fields and impedance effects from the nearby conductors. The radiation resistance $R_{r}$ determines the amount of energy that has been radiated, and the ratio of the radiation resistance to the radiation resistance plus all other losses $\mathrm{R}_{1}$ (non-radiating) determines the antenna efficiency $\eta$ or $\eta=R_{r} /\left(R_{r}+R_{l}\right)[17,20]$.

Both the transmitter and receiver also have certain characteristics features. The transmitter antenna radiates the field. Directive antennas radiate most energy in the some direction and attempt to damp down the radiation in other directions. The most important feature of a receiver antenna is the maximum signal-to-noise ratio. The radiation pattern shows the relative intensity of the radiated field in various directions from the antenna. It is a mathematical representation of the directivity of the antenna (Fig. 3). Increasing the directivity of a receiver antenna increases the signal-to-noise ratio in the receiver $[17,20]$.

Fig. (3a) presents the antenna patterns of dipole antennas in air. A quarter-wave antenna is much less directional than a full-wave antenna, and the directivity thus increases when the length of the antenna increases. However, when the length is $1>\lambda$, side-lobes appear and the directivity starts decreasing. For example, the pattern of a $1.5 \lambda$ antenna has a maximum at 45 degrees, since the directivity of longer antennas cyclically increases and decreases. The EMRE antennas are short when compared to the wavelengths in typical rock materials $\left(\varepsilon_{r}=10\right)$ at the three lower frequencies $(312.5-1250 \mathrm{kHz})$, but at the highest frequency $(2500 \mathrm{kHz})$, the longer antenna of $40 \mathrm{~m}$ is a half-wave antenna. When the permittivity of rock increases $\left(\varepsilon_{r}=40\right)$, both the long $(40 \mathrm{~m})$ and short antenna $(20 \mathrm{~m})$ are half-wave antennas at the frequencies of 1250 and $2500 \mathrm{kHz}$, respectively (Table 1). Fig. (3b, c) present a vertical dipole antenna over an imperfect surface as a function of antenna height. In Fig. (3d), the conductive medium around the antenna broadens the pattern (brown curve) and reduces the directivity [21]. Strictly speaking, the broadening effect is not a major problem at the EMRE frequencies.

When an antenna is situated near an interface across which the electrical properties change, the antenna is coupled to the interface and the radiation pattern changes when compared to the full-space situation. At the air-earth interface, the electric properties change considerably and the coupling effects may be severe, resulting in a modified radiation pattern (Fig. 3b). The Earth has a finite conductivity, which swings the maximum direction of the pattern out from the interface, and no radiation therefore occurs in the directions along the ground plane. In air, the antenna pattern of the dipole antenna is a well-known and simple symmetrical pattern (Fig. 3a), but in a conducting medium the pattern becomes broader due to the contributions from the different parts of the antenna (Fig. 3d). The refracted (along the air-earth interface) and reflected waves may interfere with the direct waves and cause significant 


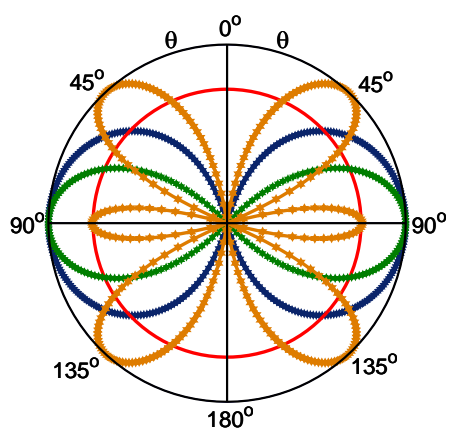

a)

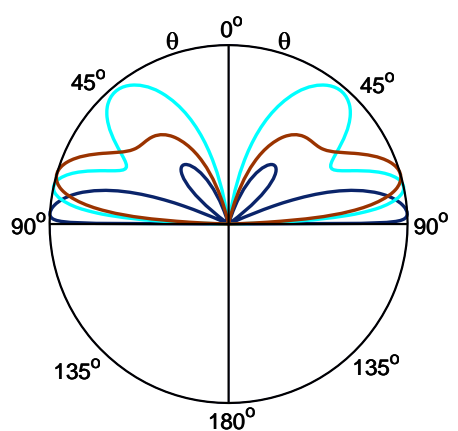

c)

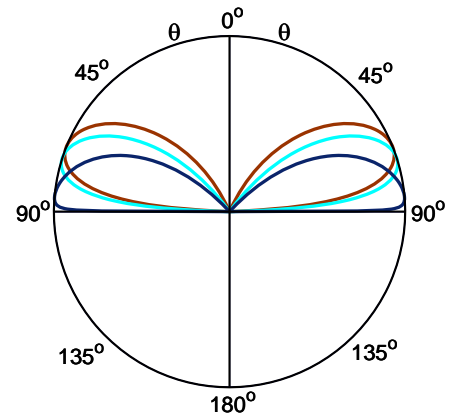

b)
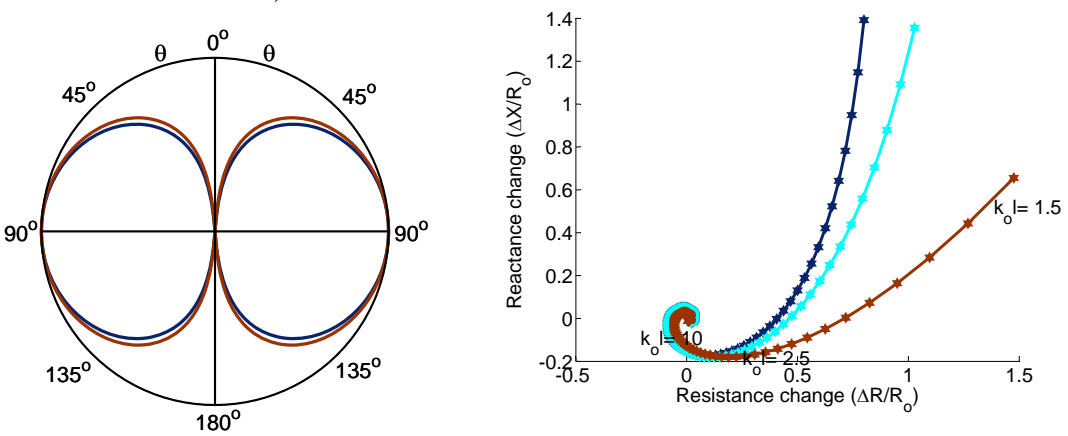

e)

Fig. (3). a) Dipole antenna patterns. The red dashed line corresponds to the circular pattern of an isotropic antenna (drawn randomly). A quarter-wave dipole $(1=0.25 \lambda)$ and full-wave dipole $(1=1.0 \lambda)$ are plotted as a blue and green line, and $1=1.5 \lambda$ as brown. $\mathbf{b}) \mathrm{A}$ dipole antenna at a height of $h=\lambda / 4$ over a conducting surface $(\mathrm{f}=1 \mathrm{MHz})$. The blue line corresponds to sea water $\left(\sigma=5 \mathrm{~S} / \mathrm{m}, \varepsilon_{\mathrm{r}}=81\right)$, the magenta line to wet granite $\left(\sigma=10^{-3} \mathrm{~S} / \mathrm{m}, \varepsilon_{\mathrm{r}}=15\right)$ and the brown line to dry granite $\left(\sigma=15^{-5} \mathrm{~S} / \mathrm{m}, \varepsilon_{\mathrm{r}}=5\right)$. c) The height is increased (h= $\lambda / 2)$. d) The effect of the conductive medium is to broaden the pattern. The antenna in air is indicated by a blue line and that in the medium $(\sigma=0.001 \mathrm{~S} / \mathrm{m}, \mathrm{f}=2.5 \mathrm{MHz}, 2 \mathrm{~L}=40 \mathrm{~m})$ is plotted in brown. The directivity decreases. e) The normalized changes in resistance and reactance as a function of antenna height $\left(\mathrm{k}_{\mathrm{o}} \mathrm{l}\right) . \mathrm{R}_{\mathrm{o}}=20\left(\mathrm{k}_{\mathrm{o}} \mathrm{dl}\right)^{2}$ is the radiation resistance of the dipole in air and $\mathrm{k}_{\mathrm{o}}=\omega^{2} \varepsilon \mu$ the free space wave number. Blue line $\left(\sigma=10 \mathrm{~S} / \mathrm{m}, \varepsilon_{\mathrm{r}}=10\right)$. Magenta line $\left(\sigma=0.01 \mathrm{~S} / \mathrm{m}, \varepsilon_{\mathrm{r}}=10\right)$. Brown line $\left(\sigma=0.0001 \mathrm{~S} / \mathrm{m}, \varepsilon_{\mathrm{r}}=10\right)$.

artefacts if they are not taken into account. The artefacts may be avoided by ensuring that the transmitter (receiver) antenna is always situated at least one dominant wavelength from the air-earth interface. Thus, when the transmitter and receiver are lowered deep enough, one would benefit from the depth gain (the effects of the air-earth interface are minimized) similarly to the situation where the dipoles are elevated above the ground surface, thereby benefitting from the height gain $[9,24]$ when the resistance and reactance of impedance decreases due to the elevation of the antenna (Fig. 3e). Earth materials have relative permittivity and conductivity values that are higher than those of air, so the wavelength is shorter along a buried metal wire than it is along a wire used in air. In air, the wavelength is equal to 300/f, where $\mathrm{f}$ is the frequency in MHz. In any medium other than free space, the wavelength is equal to $300 / \mathrm{Nf}$. The Nfactor is a function of relative permittivity and conductivity in the material. When the $\mathrm{N}$-factor has a value of 4 , it means that a half-wave dipole at $2.5 \mathrm{MHz}$ is $60 \mathrm{~m}$ long in air, but 15 $\mathrm{m}$ long when buried in rock. The earth materials surrounding the wire dipole force its length to be reduced by a factor of 4 in order to achieve the antenna characteristics that are normally attributed to a half-wave dipole. The same occurs with insulated antennas, but because the relative permittivity values of the insulation layers are $\varepsilon_{\mathrm{ri}} \sim 2,3$, the effect is not as strong.
In a dissipative medium the performance of an antenna is highly controlled by nearby conductors, because the antenna impedance is strongly influenced by the electrical properties of the medium (Fig. 3e). A bare metal wire antenna could serve as a diagnostic probe. However, when an insulated antenna is used, the diagnostic power of the antenna is highly reduced but the impedance is still a function of electrical parameters of the medium. In addition, the insulation layer changes the effect of the ambient medium. The insulated EMRE antennas are 40/20 m long. They are half-wave dipole antennas and are physically and electrically short (dimensions are small relative to the wavelength). They could be used for diagnostic purposes if an additional requirement is fulfilled. Both the medium and the insulation layer should have similar electrical properties, because the ratio of the relative permittivity of the insulation to that of the medium determines the impedance. Generally, the relative permittivity of the medium is larger than the permittivity of the insulation, but ice fulfils the requirement [22]. In water, the velocity of an electromagnetic wave is about three times lower than in common rocks. This could cause some part of the energy to be trapped in the borehole and result in the waves propagating as guided waves along the borehole with the velocity of the surrounding rock, thus being able to radiate energy along the borehole. However, theoretical studies suggest that the waveguide effects are only significant as long as the dominant wavelength of an 
electromagnetic wave is less than 5-10 times the diameter of the borehole [23]. In low resistivity $(1 \Omega m)$ water, the dominant wavelengths are from $10 \mathrm{~m}(100 \mathrm{kHz})$ to $1.2 \mathrm{~m}$ $(6000 \mathrm{kHz})$. In more resistive borehole water $(250 \Omega \mathrm{m})$, the wavelengths increase substantially, being from $15 \mathrm{~m}(100$ $\mathrm{kHz})$ to $5.5 \mathrm{~m}(6000 \mathrm{kHz})$. Thus, in the $312.5-2500 \mathrm{kHz}$ band, the waveguide phenomenon is not significant and may be bypassed in typical boreholes (46-76 $\mathrm{mm}$ in diameter) in the EMRE frequency band.

The important electrical earth parameters that have a strong effect on the antenna are conductivity and dielectric permittivity. Normally, these parameters are complex quantities with conductivity in the form of $\sigma=\sigma^{\prime}+i \sigma^{\prime \prime}$ and permittivity $\varepsilon=\varepsilon^{\prime}+i \varepsilon^{\prime \prime}$. Conductivity is the response of free charges to an applied field. It describes the ability of the material to pass free charges. The relaxation phenomenon is small in conductors. At low frequencies, the charges respond instantaneously to an applied field. Thus, the current varies in-phase with the electric field and the conductivity can be represented by a static value $(D C)$. However, at much higher frequencies the relaxation times $\tau$ are large, resulting in an out-of-phase component $\left(\sigma^{\prime \prime}\right)$. The permittivity is a measure of the response of bound charges to an applied field (polarization). It describes the ability of a material to store or release energy. The relaxation phenomenon can also be an important factor in the EMRE frequencies. In the relaxation phenomenon, the time-dependent displacement mechanism acts at different rates to an applied electric field. Below the relaxation frequency $\left(f_{r}=1 / \tau\right)$, the charges quickly react to an applied field and remain in phase with its changes. As frequencies increase, the polarization lags behind the changing field, resulting in an out-of-phase component $\left(\varepsilon^{\prime \prime}\right)$. The real component $\varepsilon^{\prime}$ of permittivity is related to the energy storage effects and the complex component $\varepsilon$ " to the losses in polarization and displacement. The relaxation mechanisms can be divided into two groups: bound charge effects $(e . g$. electronic, atomic, dipolar) and free charge effects (e.g. Maxwell-Wagner) [4]. Bound charge polarization is related to much higher frequencies than the EMRE band and can be ignored. However, the Maxwell-Wagner effect, where free ionic charges can be trapped in porous water and on grain surfaces, can already be notable from a frequency level of $100 \mathrm{kHz}$ [2]. The parameters are expressed in Helmholtz equations $\left(e^{-i \omega t}\right)$ in the following combination $[9,11,13]$

$\widehat{\varepsilon}=\sigma-\mathrm{i} \omega \varepsilon$

Taking into account the complex representations and using Eq. 1, the effective conductivity and permittivity, which are the directly measurable quantities, can be written as

$\sigma_{e}=\sigma^{\prime}+\omega \varepsilon^{\prime \prime} \varepsilon_{e}=\varepsilon^{\prime}-\sigma^{\prime \prime} / \omega$

According to $E q .2, \sigma^{\prime}$ and $\varepsilon^{\prime \prime}$ both produce a current that varies in phase with the applied electric field, and $\sigma^{\prime \prime}$ and $\varepsilon^{\prime}$ both produce a current out of phase with the electric field. Generally, the magnetic permeability is real and can be expressed as $\mu=\mu_{0} \mu_{\mathrm{r}}$, where $\mu_{0}=4 \pi * 10^{-7} \mathrm{H} / \mathrm{m}$. The relative permeability $\mu_{\mathrm{r}}$ is equal to unity in most rocks, or $\mu=\mu_{0}$ is a valid assumption. The wave number $\mathrm{k}$ is an important parameter that binds the conductivity, permittivity, permeability and frequency. The wave number can be expressed as $\mathrm{k}=2 \pi / \lambda$, where $\lambda$ is the wavelength of an electromagnetic wave. Generally, the wave number is a complex quantity:

$$
k=\beta+i \alpha=\begin{array}{ll}
\omega \sqrt{\mu \varepsilon_{e}} \sqrt{\left(1+i \cdot \operatorname{loss}_{e}\right)} & \varepsilon_{\mathrm{e}}>0 \\
\sqrt{i \cdot \omega \mu \sigma_{e}} & \varepsilon_{\mathrm{e}}=0
\end{array}
$$

where $\beta$ is the phase constant $(\mathrm{rad} / \mathrm{m}), \alpha$ is the attenuation constant $(\mathrm{Np} / \mathrm{m}), \quad \operatorname{loss}_{\mathrm{e}}$ is the loss tangent and $\operatorname{loss}_{e}=\sigma_{e} / \omega \varepsilon_{e}$. The relative permittivities of most earth materials are positive and larger than 1 , or $\varepsilon_{\mathrm{r} 1} \geq 1$ [22]. Thus,

$\beta=\omega \sqrt{\frac{\mu \varepsilon_{e}}{2}} \sqrt{\sqrt{1+\operatorname{loss}_{e}^{2}}+1}>\alpha=\omega \sqrt{\frac{\mu \varepsilon_{e}}{2}} \sqrt{\sqrt{1+\operatorname{loss}_{e}^{2}}-1}$

The Q-factor is equal to the inverse value of the loss tangent. The behaviour of the Q-factor is closely related to the behaviour of the field in a medium where the field exists $[7-8,25]$. It is thoroughly treated in a paper by Korpisalo [14]. Fig. (4) presents the attenuation and phase constants as a function of frequency. The cut-off frequencies determine the frequencies when a quasi-static condition is valid or $\alpha \approx$ $\beta$. When the cut-off frequency has been reached, both the attenuation and phase curve deviate from the quasi-static curve (black) and the field propagates as waves through the medium. In a low resistive medium $(\rho=100 \Omega m)$, the cutoff frequency for quasi-static condition is at $\approx 1000 \mathrm{kHz}$, while in a highly resistive medium $(\rho=10000 \Omega \mathrm{m})$ the quasi-static limit is at $10 \mathrm{kHz}$ (Fig. 4a). The effect of increasing the relative permittivity is to decrease the cut-off frequency. In a low resistive medium $(\rho=100 \Omega \mathrm{m})$, the quasi-static condition is valid up to $100 \mathrm{kHz}$, while in a highly resistive medium $(\rho=10000 \Omega m)$ the quasi-static condition can reach up to $10 \mathrm{kHz}$ (Fig. $4 \mathbf{b}$ ).

In air, the calculation of the antenna properties is quite straightforward. The average flow of power through a sphere surrounding the antenna is needed when determining the radiation resistance and gain. In a dissipative medium, the calculations of the antenna properties become more complicated and no analytical solutions exist. Numerical methods must therefore be taken into use. However, it is possible to estimate, for instance, the power density using the same methods as in a non-conducting medium, but the electrical parameters of the medium must be treated like complex quantities and the average power is strongly dependent on the distance $\mathrm{R}$ from the antenna. In air, the same power flows through any sphere. In a dissipative medium, there is a large difference in energy flow through different spheres, especially in the vicinity of the dipole. Thus, it is not meaningful to estimate the values of antenna parameters (e.g. antenna pattern, gain, directivity) in a dissipative medium, or the distance from the source must be included in the definitions of these parameters [21]. 


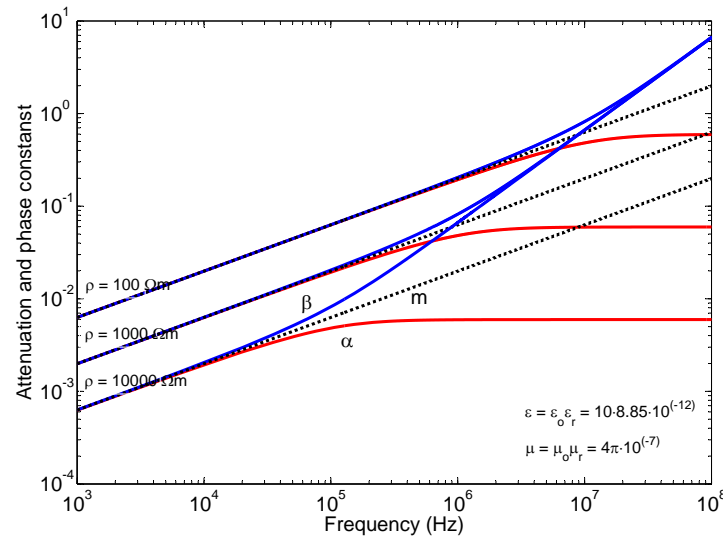

a)

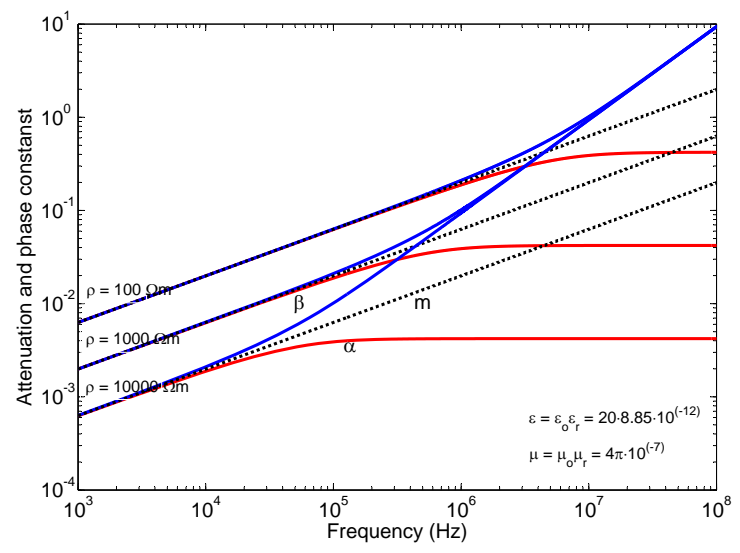

b)

Fig. (4). a) The attenuation $(\alpha)$ and phase $(\beta)$ constants in a dissipative medium with $\varepsilon_{\mathrm{r}}=10$ and $\rho=100,1000$ and $10000 \Omega \mathrm{m}$. b) The phase and attenuation constants in a dissipative medium with $\varepsilon_{\mathrm{r}}=20 . \alpha$ is the attenuation (red lines) as Nepers/m and $\beta$ is the phase constant (blue lines) as $\mathrm{rad} / \mathrm{m}$. The $\mathrm{m}$ curve is valid when the attenuation constant equals the phase constant or $\alpha \approx \beta$ (black curves).

\section{NUMERICAL STUDIES AND RESULTS}

In this paper, a commercial simulation package FEKO [19] is utilized for the numerical modelling of characteristics of the insulated antennas. FEKO is an electromagnetic simulation package developed by EM Software and Systems and runs in the frequency domain. The Methods of Moments $(M o M)$ is a full wave solution of Maxwell's integral equations in which only the structures in question are discretized, not free space. The shapes of objects can be arbitrary (Fig. 5). The preliminary step is to determine the electric currents on the conducting surfaces and the equivalent electric and magnetic surface currents on the surface of dielectric solids. The electromagnetic fields and, for instance, the antenna impedances are then calculated from the current distributions.

Generally, an antenna tuner (ATU) would match the transmitter generator or source impedance to the load (antenna) impedance. A mismatch is usually caused when a non-resonant antenna is used. The radiated power is a complex quantity and consists of an active and a reactive component. The reactive power component oscillates in the near field of the antenna. To cancel the complex component and to make the antenna resonant at the operating frequency, an inductor or a capacitor can be connected to the antenna system [26]. These components are passive and they do not change the active power, but they change the reactive power in the system to ensure that the reactance is zero. In the EMRE system, the ATU is lacking. In addition, open-end terminations $\left(Z_{s}=\infty\right)$ are used. Thus, high voltage standing wave ratios $(V S W R)$ are evident [18]. When looking at the complete circuit (e.g. transmitter, antenna) and its functionality, the borders with the outer space are of greatest interest. Thus, when isolating the circuit and defining a limited number of ports, where it interacts with the outer space, simple notations (scattering parameters) can be used

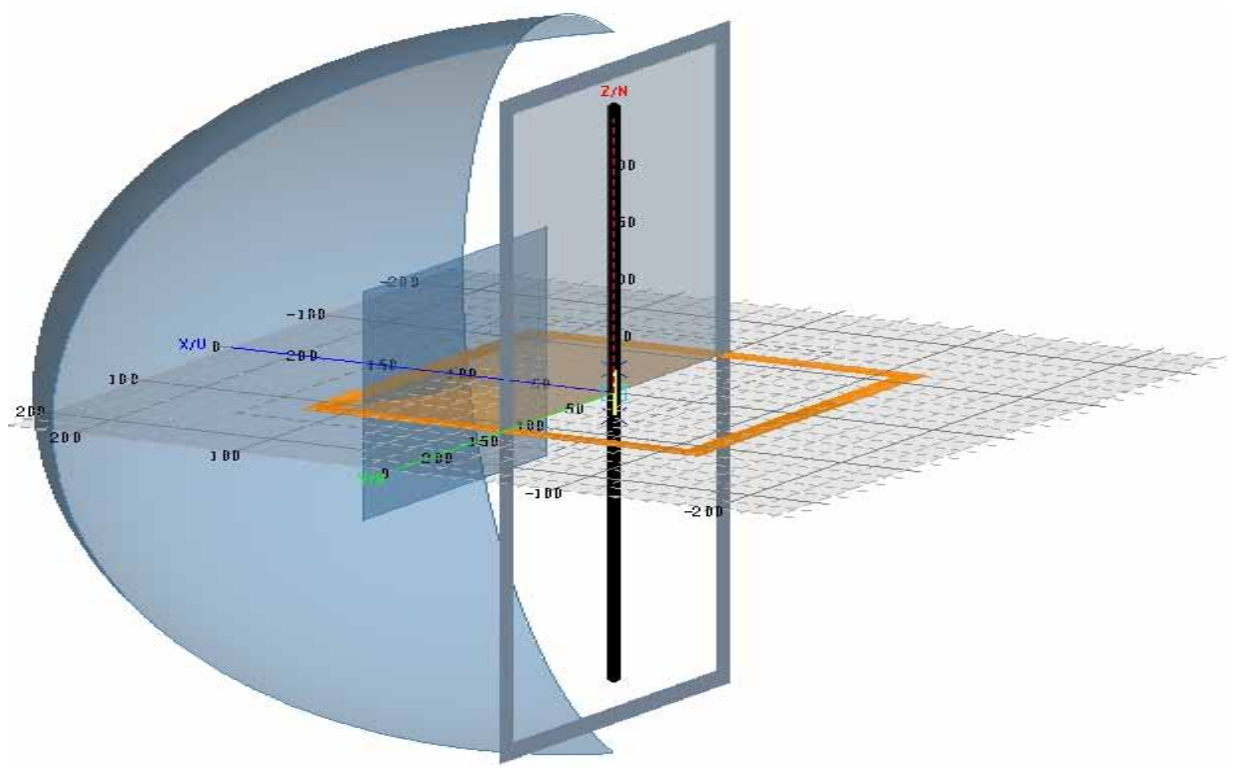

Fig. (5). The numerical FEKO model. The yellow bar represents the antenna. An insulated antenna $(-20<\mathrm{z}<20$, yellow) is centred in a water-filled borehole $(-250<\mathrm{z}<250$, diameter $76 \mathrm{~mm}$, green tube). The symmetry planes make calculations faster. Electric symmetry: orange $\mathrm{z}=0$ plane and magnetic symmetry: $\mathrm{x}=0$ plane. 
The components of impedance and scattering parameters $\mathrm{s}_{11}$ of an antenna

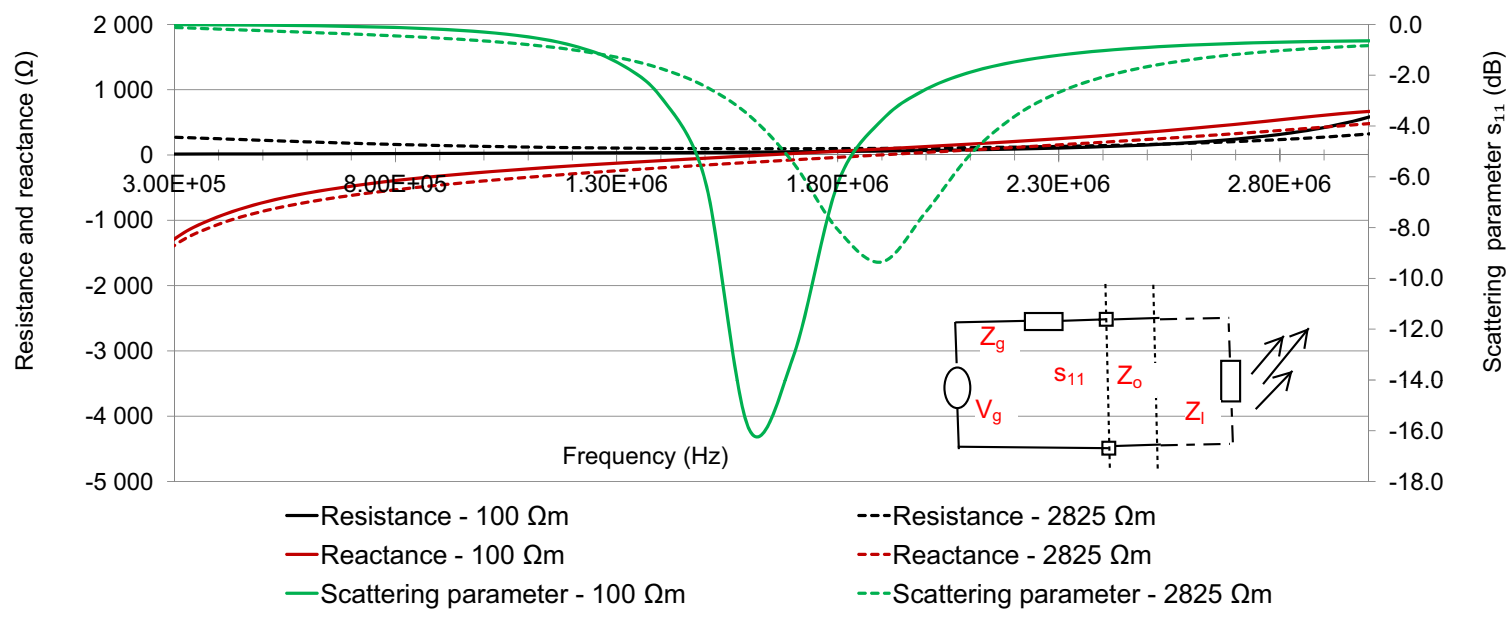

Fig. (6). The resistances and reactances of the impedance and the scattering parameter $s_{11}$ an insulated dipole antenna. The length of antenna is $40 \mathrm{~m}$ and the relative permittivity of insulation is $\varepsilon_{\mathrm{r}}=1$. The rock resistivity is $100-2825 \Omega \mathrm{m}$ and the relative permittivity $\varepsilon_{\mathrm{r}}=8$. The lumped port impedance is $50 \Omega$. A schematic representation of an antenna is shown.

to describe its functionality. The scattering parameter $\mathrm{s}_{11}$ defines the relationship between the reflected and incident voltage in the input port of the antenna [7]. According to the schematic representation in Fig. (6), the antenna is well matched when at first the output impedance $\mathrm{Z}_{\mathrm{G}}$ of the source is equal to the characteristic impedance of the cable $Z_{0}$ to ensure that all of the source's output power goes to the cable and is not reflected back to the source. Secondly, the input impedance of the electric load $Z_{L}$ must be equal to the characteristic impedance, so that all of the power goes the load rather than being reflected from the load [7, 17, 18]. In Fig. (6), the resistances (black curves) and reactances (red curves) and scattering parameters (green curves) of an insulated dipole antenna are presented.

In a fixed-length antenna, the reactance alternates cyclically (capacitive-inductive-capacitive, etc.) as a function of frequency, and the zero values of reactances correspond to the different resonance positions. The resistive component of the antenna impedance should equal $50 \Omega$ (well matched) with a zero reactive component (Fig. 6). This occurs at $\sim 1.6 \mathrm{MHz}$, which is the first resonance point of the antenna at a frequency of $\sim 1.6 \mathrm{MHz}$ when $\mathrm{s}_{11}$ is $\sim-16 \mathrm{~dB}$, meaning that only $\sim 3 \%$ is reflected and $\sim 97 \%$ is delivered to the antenna, embedded in a low resistive medium $(100 \Omega \mathrm{m})$. In a more resistive medium $(2825 \Omega \mathrm{m})$, the first resonance occurs at a frequency of $\sim 1.9 \mathrm{MHz}$ when $\mathrm{s}_{11}$ is $\sim-9 \mathrm{~dB}$, meaning that $\sim 11 \%$ is reflected and $\sim 89 \%$ is delivered to the antenna. $\left(s_{11}(d B)=20 \cdot \log _{10}\left(s_{11}(\right.\right.$ lin $\left.)\right)$ or $s_{11}($ lin $)=10^{\wedge-}$ $\left.{ }_{11}^{(d B / 20)}=\sqrt{ }\left(P_{r e f} / P_{f w}\right)\right)$.

One of the distinctive features of the EMRE system is that both antennas are situated in deep boreholes, which are usually water-filled. They are classified as insulated antennas due to the presence of insulation layers around the central conductors of the antennas. When a metal wire antenna (probe dipole) resides in even a moderate resistive material, the current in the antenna will diminish along the antenna axis, because the surrounding material acts as a complementary path for the current. As the current in the antenna is very sensitive to the surrounding material, a metal wire could be used as a diagnostic probe to investigate the electric parameters of the borehole vicinity. In general, the antennas are highly insulated. Depending on its thickness, the insulation will not only prevent the leakage of conducting charges from the antenna but will also greatly reduce the sensitivity of the entire current distribution to the electrical properties of the ambient medium [22]. The current distributions of an insulated and a metal wire antenna were compared in a water-filled borehole, where the relative permittivity of rock is $\varepsilon_{\mathrm{rr}}=8.0$ and that of the insulation $\varepsilon_{\mathrm{ri}}=$ 1.0. Water has a high relative permittivity of $\varepsilon_{\mathrm{rw}}=81$. The resistivity of rock is $\rho_{\mathrm{r}}=10000 \Omega \mathrm{m}$ and that of water $\rho_{\mathrm{w}}=1$, $1000 \Omega \mathrm{m}$. The antennas are $40 \mathrm{~m}$ long. The FEKO model of a buried insulated antenna is illustrated in Fig. (5). When the borehole water is conductive, i.e. $\rho_{w}=1 \Omega \mathrm{m}$, it effectively serves as a complementary path for the current in the metal wire antenna (Fig. 7).

In Fig. (8), the resistivity of borehole water has been increased to a value of $\rho_{w}=1000 \rho m$, resulting in similar current waveforms. Thus, the water layer has lost its power to serve as a complementary path for the current. Both antennas are near resonance: the metal wire antenna has just passed it, but the insulated antenna is still short.

Normally, the Methods of Moments (MoM), for instance, is used to change the integral equations into matrix equations, when metal wires are of interest. However, after King [22], a generalized transmission line model (coaxial line model) can be used to eliminate the tedious integrals (Figs. 9, 10). It is assumed that the inequalities $\left|k_{2} b\right|<<1$, $\left|k_{3} c\right|<<1$ and $\mathrm{h}>\mathrm{c}>\mathrm{b}$ are satisfied, or the layers are narrow compared to the wavelengths.

The wave numbers associated to the different layers are $k_{1}=\left(i \omega \mu \sigma_{1}\right)^{1 / 2}=(1+i)\left(\frac{\omega \mu \sigma_{1}}{2}\right)^{1 / 2}$ conductor 


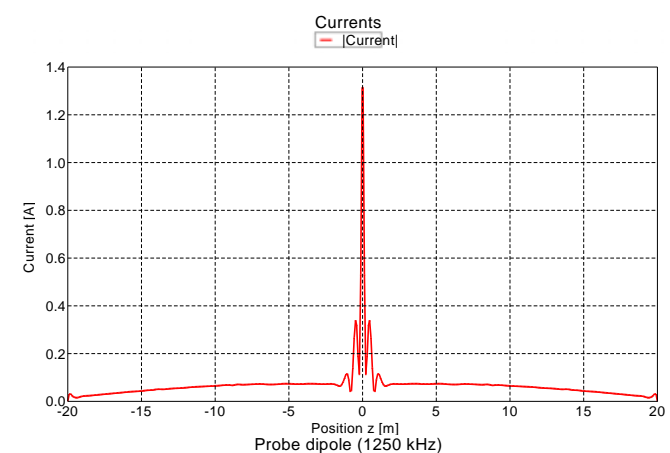

a)

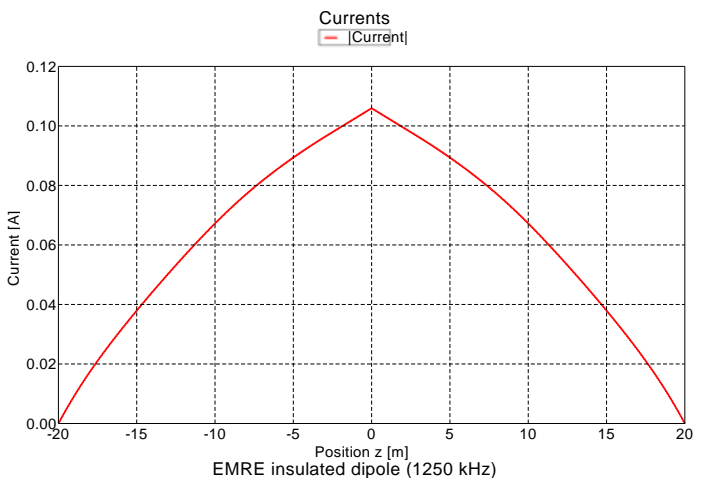

b)

Fig. (7). The frequency is $1250 \mathrm{kHz}$ and the resistivity of borehole water is $\sigma_{\mathrm{w}}=1 \Omega \mathrm{m}$. a) The water is a complementary current path in a metal wire antenna and the current is concentrated near the feeding point (having a peak value of 1.3 A). b) An insulated antenna is approaching its first resonance length and the current waveform becomes sinusoidal (current peak value is $\sim 0.1 \mathrm{~A}$ ).

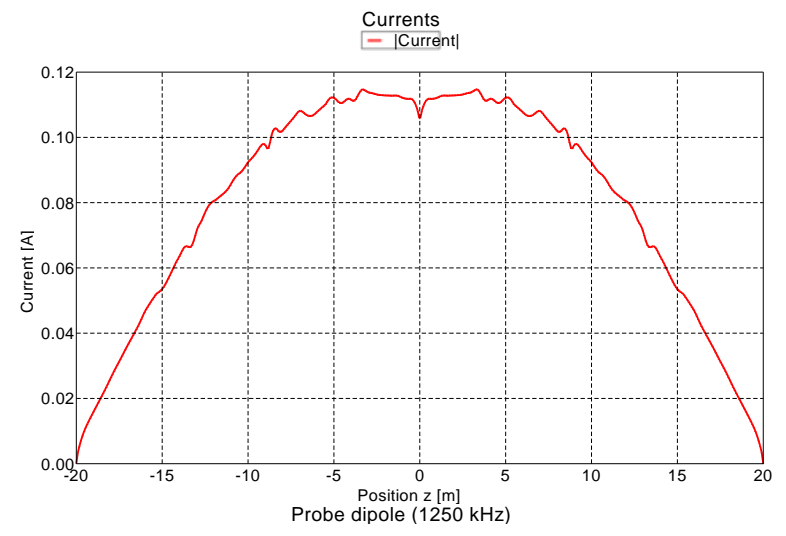

b)

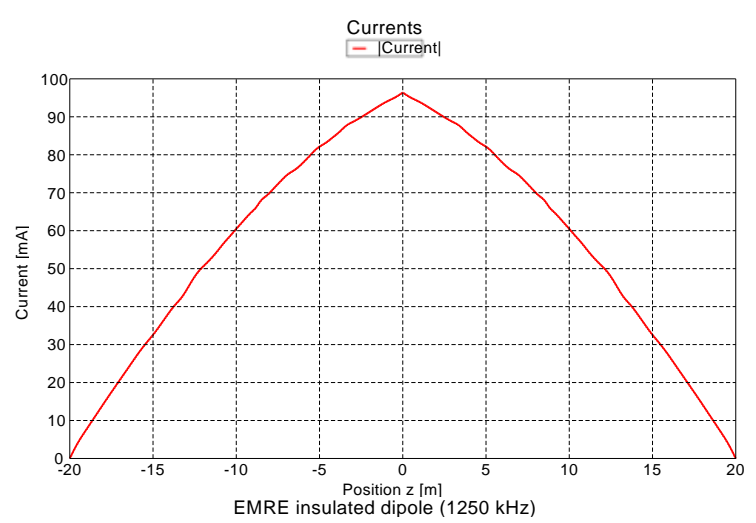

Fig. (8). The frequency is $1250 \mathrm{kHz}$ and the resistivity of borehole water is $\rho_{\mathrm{w}}=1000 \Omega \mathrm{m}$. a) A metal wire antenna has passed its half-wave length (current peak value is $0.12 \mathrm{~A}$ ). b) An insulated antenna is still short compared to the wavelength and the current waveform becomes more sinusoidal. (Current peak value is $0.1 \mathrm{~A}$ ).

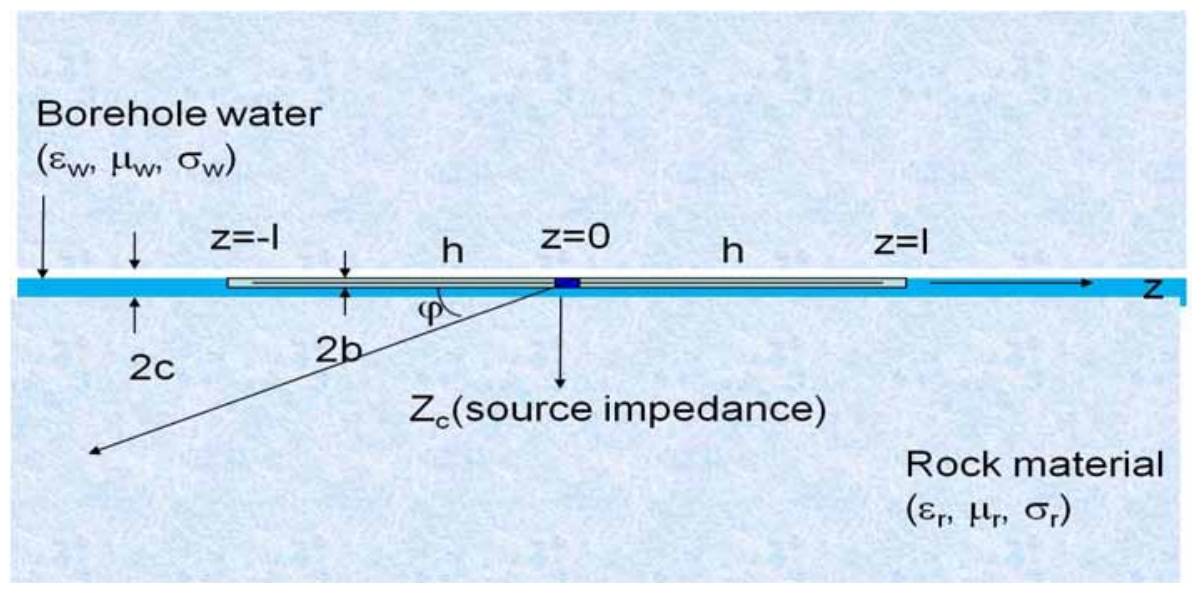

Fig. (9). An insulated one-layer antenna in a water-filled borehole (not to scale).

$$
\begin{aligned}
& k_{2}=\omega\left(\mu \varepsilon_{2}\right)^{1 / 2}\left(1+\frac{i \sigma_{2}}{\omega \varepsilon_{2}}\right)^{1 / 2} \text { first insulator } \\
& k_{3}=\omega\left(\mu \varepsilon_{3}\right)^{1 / 2}\left(1+\frac{i \sigma_{3}}{\omega \varepsilon_{3}}\right)^{1 / 2} \text { second insulator }
\end{aligned}
$$

$$
k_{4}=\left(i \omega \mu \sigma_{4}\right)^{1 / 2}=(1+i)\left(\frac{\omega \mu \sigma_{4}}{2}\right)^{1 / 2} \text { conductor }
$$

It has been shown by King [22] that even if the antenna has two or more insulation layers $\left(\varepsilon_{2}\right.$ and $\left.\varepsilon_{3}\right)$, it is possible to determine the effective relative permittivity as 


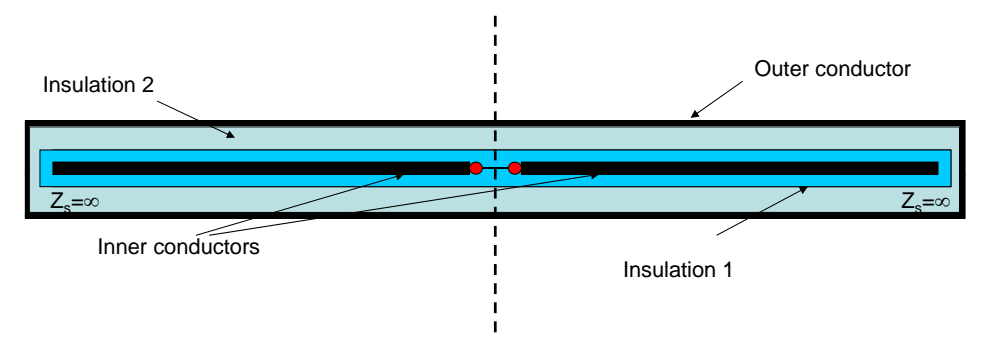

Fig. (10). Transmission line model of an insulated dipole antenna with two identical sections [22]. The outer and inner conductor (black) are metal regions.

$\varepsilon_{e f f, r}=\varepsilon_{2}\left[\frac{\ln (c / a)}{\ln (b / a)+\eta_{23}^{2} \ln (c / b)}\right]$,

where $a$ is the conductor radius, $b$ is the radius of the first insulator and $c$ is the radius of the second insulator. Factor $\eta_{23}$ is defined by $k_{2} / k_{3}$. According to King [22], the current of the open-end section of a thin antenna driven by voltage $\mathrm{V}_{\mathrm{o}}$ can be assumed to be sinusoidal

$I(z)=I_{o} \frac{\sin k_{h}(h-|z|)}{\cos k_{h} h}=\frac{i * V_{o}}{2 Z_{c}} \frac{\sin k_{h}(h-|z|)}{\cos k_{h} h}$

and the corresponding input impedance of the antenna is given by

$Z_{\text {in }}=-i * 2 Z_{c} \cot \left(k_{h} * h\right)$

where $Z_{c}$ is the characteristic impedance of the transmission line and $\mathrm{k}_{\mathrm{h}}$ is the complex wave number of the transmission line $[18,27,28]$. As can be seen from Eq. 8, the antenna serves as a transformer. In the WKG model (Wu-King-Giri), the impedance of the transmission line consists of three serial impedances and a shunt impedance. The model is accurate to $\left|k_{4}^{2}\right|>16\left|k_{2}^{2}\right|$, which greatly restricts its usefulness [22, 28]. Strictly, the model could only be used when the antenna is embedded in water [28]. The CHEN model (Chen and Warne) introduced the admittance of the ambient medium to the model. The impedance consists of three serial impedances and two shunt impedances. The transmission line wave number $\mathrm{k}_{\mathrm{h}}$ is given by Gouws [28] and Sato [27] as

$k_{h}=k_{2}\left[\frac{k_{4}^{2}\left\{H_{0}^{(2)}\left(k_{4} b\right)+k_{4} b \ln (b / a) H_{1}^{(2)}\left(k_{4} b\right)\right\}}{k_{2}^{2} H_{0}^{(2)}\left(k_{4} b\right)+k_{4}^{2} k_{4} b \ln (b / a) H_{1}^{(2)}\left(k_{4} b\right)}\right]^{1 / 2}$

where $H_{n}^{(2)}$ is the Hankel function of second kind of order $\mathrm{n}$. The CHEN model is assumed to be accurate, when $\left|k_{4}^{2}\right|>2\left|k_{2}^{2}\right|$ is satisfied, and the model can be used in both conductive and dielectric conditions. The water-filled borehole must be excluded in both of these transmission line models ( $W K G$ and $C H E N$ ), because the wave number of water is much larger than that of ambient rock and cannot be introduced [22, 28]. Thus, the outer conductor of the transmission line model must correspond to the ambient rock.

In the FEKO model, a lumped port element is used as a voltage source (Fig. 5) [29]. The conductive arms are assumed to be perfectly electric conductors (PEC). Using the
PEC assumption, only the surface of a conductor must be discretized in FEKO/MoM. The comparisons of the different methods (WKG/CHEN/FEKO) were performed with the water layer removed from the model in Fig. (9). The ambient medium had resistivity values of $\rho_{\mathrm{r}}=100,5555$ and 11000 $\Omega \mathrm{m}$, and relative permittivity $\varepsilon_{\mathrm{rr}}=8,20,32$, and that of the antenna insulation $\varepsilon_{\mathrm{ri}}=3$. In Figs. (11-13), the FEKO results are plotted in dark blue, WKG results in red and CHEN results in green.

In Fig. (11), the relationship between the wave numbers $\left(\left|\mathrm{k}_{4}^{2}\right| /\left|\mathrm{k}_{2}^{2}\right|\right)$ is low, being $\sim 1.6$. In the WKG model, it should be >16; thus, the WKG model (red curves) suffers from the disadvantageous conditions. The correspondence between the FEKO and CHEN models is good in the middle band of EMRE frequencies, but becomes worse when the resistivity of the external medium and frequency increase. The first resonance occurs at $\sim 920 \mathrm{kHz}$ in all models in a lowresistive medium $\left(\rho_{r}=100 \Omega m\right)$, but when the resistivity of rock increases, the first resonance point of the FEKO and CHEN models $(\sim 1.4 \mathrm{MHz})$ clearly differs for the result of the WKG model $(\sim 920 \mathrm{kHz})$. The increase in the relative permittivity of the rock $\left(\varepsilon_{\mathrm{ri}}=20\right)$ has a clear effect on all models, but it is quite evident that the WKG model still suffers from the low wave number relationship of $~ 2.6$ (Fig. 12). The first resonance points of the FEKO and CHEN models have moved towards lower frequencies and occur at $\sim 1 \mathrm{MHz}$. The favourable frequency band for the EMRE measurements has decreased, but the correspondence between all models has become better across the whole frequency band. The increase in rock permittivity $\left(\varepsilon_{\mathrm{ri}}=32\right)$ increases the wave number ratio $(\sim 3.3)$, and the

correspondence between all models is better across the whole frequency band (Fig. 13). In the FEKO and CHEN models, the characteristic effect of increasing the permittivity value of rock is to move the first resonance point towards lower frequencies (wavelength increases). As a summary, the analytical models provide a simple means to examine the functioning of an antenna in rock. The models are easily implemented, for instance in Matlab. The results of the CHEN model are comparable with the numerical FEKO model, but the WKG model suffers from the electrical properties of rocks and its use is not justified.

The control tubes of the EMRE system are slim (diameter $36 \mathrm{~mm}$ ), and EMRE can therefore be used in almost all boreholes. It is also usual that the boreholes are water-filled. Using the FEKO model (Figs. 5, 9), the effect of the water layer on the antenna impedance is estimated in a borehole $76 \mathrm{~mm}$ in diameter. In the model, the relative permittivity of rock has the values of $\varepsilon_{\mathrm{rr}}=8,32$, the antenna 

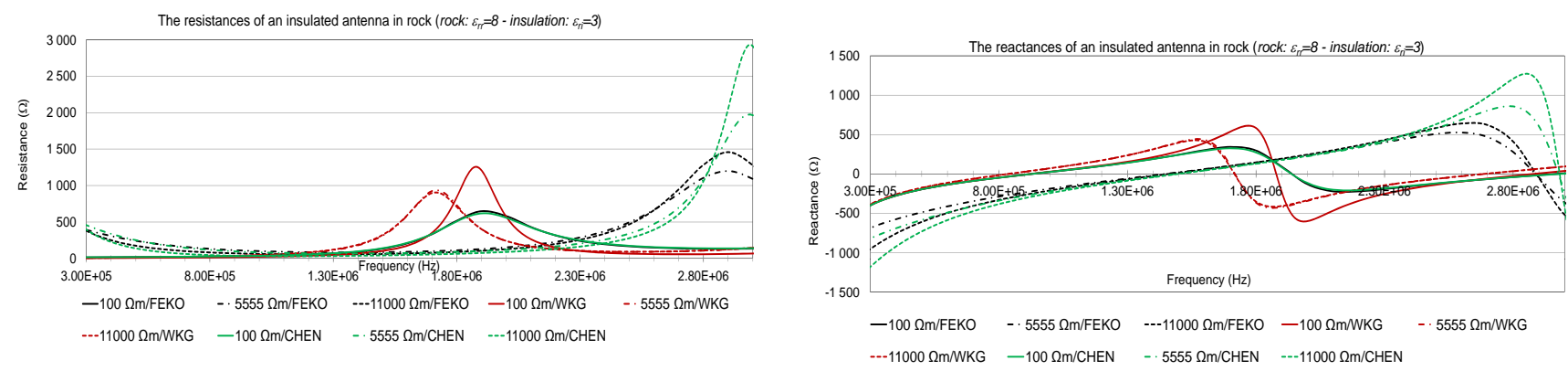

a)

b)

Fig. (11). The numerical impedances of an insulated antenna in rock $\left(\rho_{\mathrm{r}}=100,5555,11000 \Omega \mathrm{m}\right)$ by FEKO/WKG/CHEN. Relative permittivities $\varepsilon_{\mathrm{rr}}=8$ for rock and $\varepsilon_{\mathrm{ri}}=3$ for insulation. a) Resistance. b) Reactance.

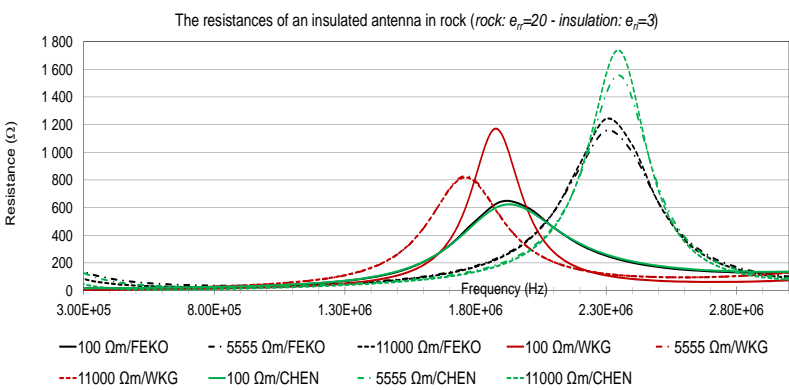

a)

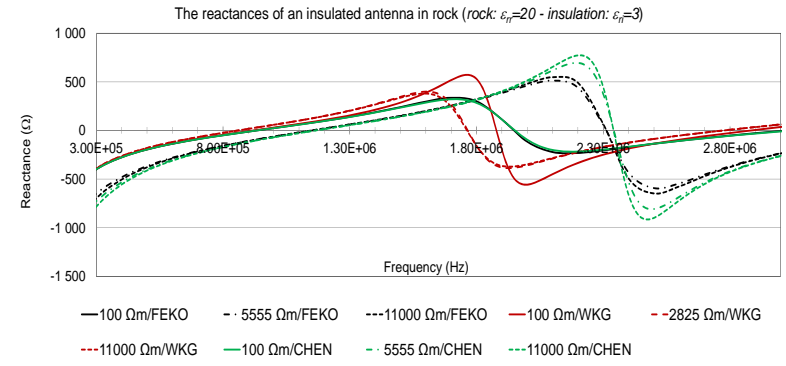

b)

Fig. (12). The numerical impedances of an insulated antenna in rock $\left(\rho_{\mathrm{r}}=100,5555,11000 \Omega \mathrm{m}\right)$ by FEKO/WKG/CHEN. Relative permittivities $\varepsilon_{\mathrm{rr}}=20$ for rock and $\varepsilon_{\mathrm{ri}}=3$ for insulation. a) Resistance. b) Reactance.

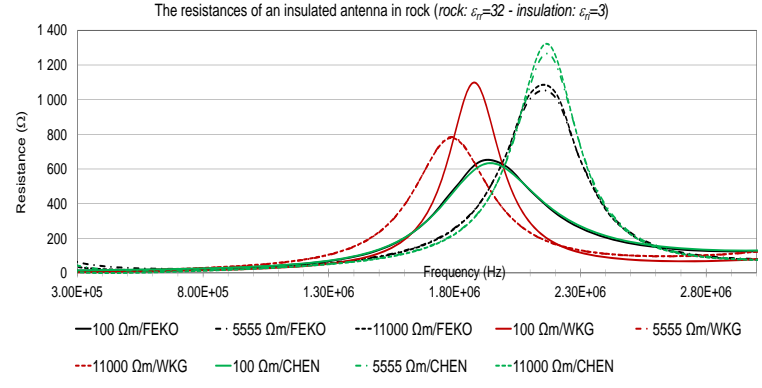

a)

Fig. (13). The numerical impedances of an insulated antenna in rock $\left(\rho_{\mathrm{r}}=100,5555,11000 \Omega \mathrm{m}\right)$ by FEKO/WKG/CHEN. Relative permittivities $\varepsilon_{\mathrm{rr}}=32$ for rock and $\varepsilon_{\mathrm{ri}}=3$ for insulation. a) Resistance. b) Reactance.

insulation $\varepsilon_{\mathrm{ri}}=3$ and water $\varepsilon_{\mathrm{rw}}=81$. Rock resistivity has values of $\rho_{\mathrm{r}}=100,1462,2825$ and $11000 \Omega \mathrm{m}$, and that of the borehole water, $\rho_{\mathrm{w}}=1,500$, and $1000 \Omega \mathrm{m}$. Both components of the antenna impedance (resistance and reactance) are presented in Figs. $(\mathbf{1 4}, \mathbf{1 5})$. In Fig. (14), the water layer has insignificant effects in the middle frequencies of the EMRE band when low resistive rock (100 $\left.\Omega m, \varepsilon_{r r}=8\right)$ is concerned. In a more resistive environment $\left(\rho_{r}>>100 \Omega m\right)$ and at the higher frequencies $(>2 \mathrm{MHz})$, the resistances and reactances clearly deviate from the others when the resistivity of water increases. The first resonance points move upward $(\sim 1.1 \mathrm{MHz} \rightarrow \sim 1.6 \mathrm{MHz})$ when the rock resistivity increases. An increase in the external permittivity $\left(\varepsilon_{r r}=8 \rightarrow \varepsilon_{r r}=32\right)$ makes the curves diverge less (Fig. 15).

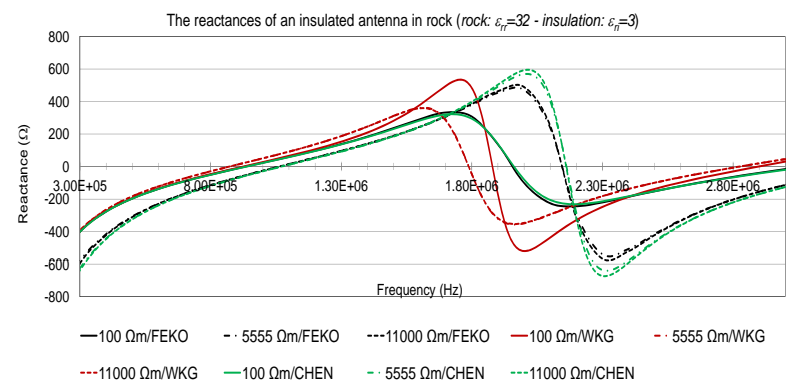

No movement of first resonance points occurs. Thus, the water layer appears to have a profound effect on the resistances and reactances after the middle band of the EMRE frequencies $(>1.6 \mathrm{MHz})$ when the permittivity of rock is $\varepsilon_{r r}=8$, but the effect is smaller when permittivity increases $\left(\varepsilon_{r r}=32\right)$. In the lower band $(<0.5 \mathrm{MHz})$, the resistances and reactances increase when the resistivity of rock increases, but the increase in permittivity $\left(\varepsilon_{r r}=8 \rightarrow \varepsilon_{r r}\right.$ = 32) cancels out the divergence (Fig. 15). According to these results, the highest frequency $(2500 \mathrm{kHz})$ seems to be problematic.

Generally, the antenna lies against the borehole wall. Models in Fig. (16) can be used to examine how the input impedance $\mathrm{Z}$ of the antenna $(Z=R+i \cdot X ; R$ is the resistance 


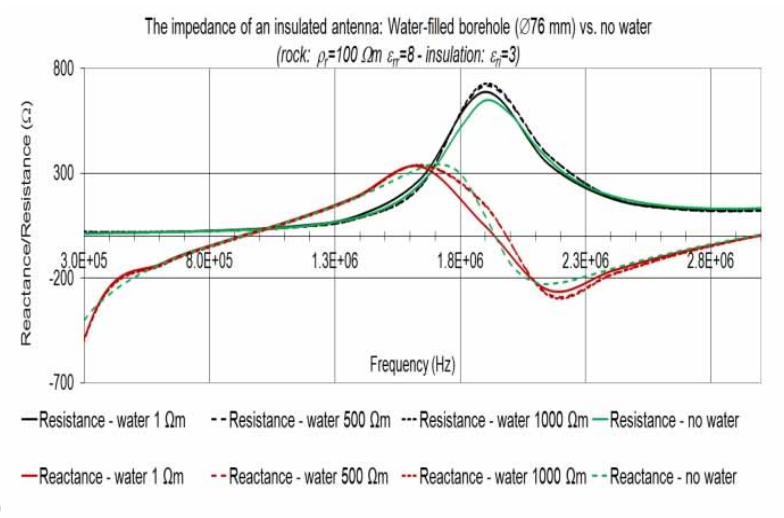

a)

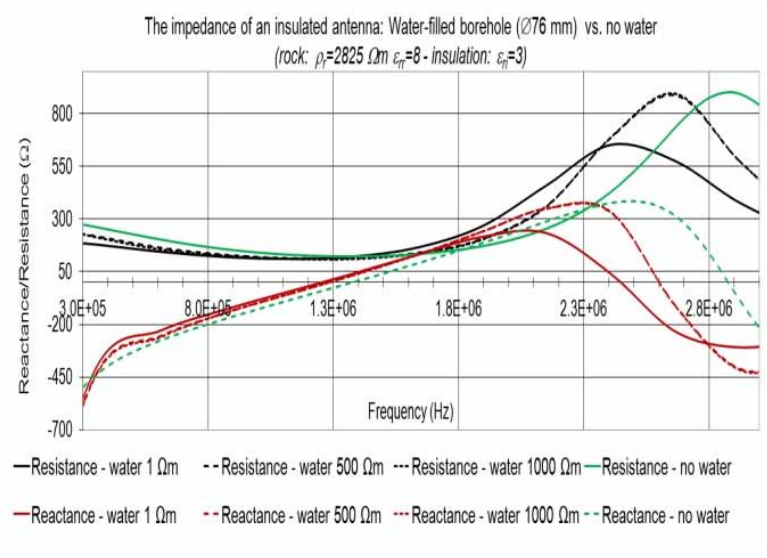

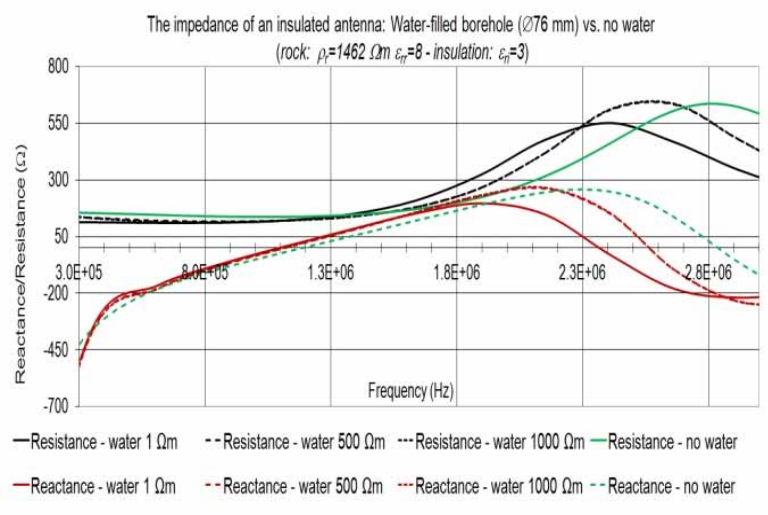

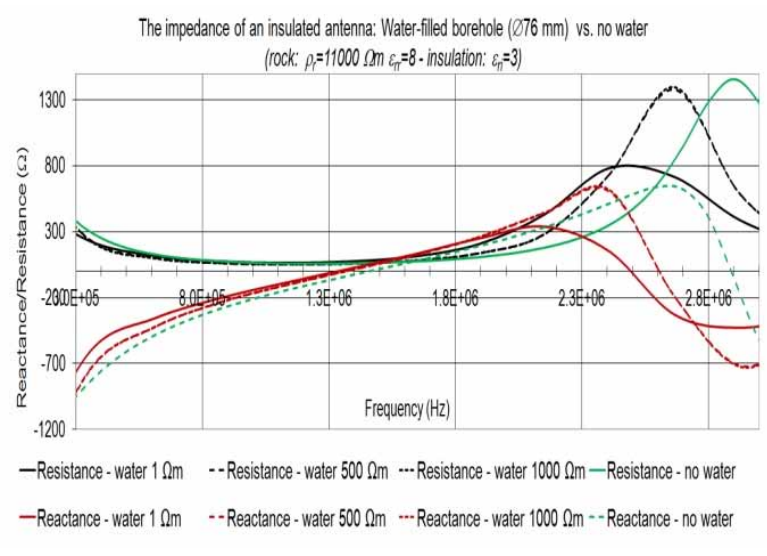

Fig. (14). Comparison of a water-filled borehole and one without water. The input impedance of an antenna. Relative permittivities $\varepsilon_{\mathrm{rr}}=8$ for rock and $\varepsilon_{\mathrm{ri}}=3$ for insulation. Resistivities of the external medium: a) $\left.\left.\left.100 \Omega \mathrm{m}, \mathbf{b}\right) 1462 \Omega \mathrm{m}, \mathbf{c}\right) 2825 \Omega \mathrm{m}, \mathbf{d}\right) 11000 \Omega \mathrm{m}$. The solid lines represent resistances and the dashed lines reactances. The green lines represent the situation without a water layer.

and $X$ the reactance) and the scattering parameter $\mathrm{s}_{11}$ change when the antenna is moved away from borehole's midpoint closer to the borehole wall. In first model, the antenna is located in a central position and the outer surface is $30 \mathrm{~mm}$ from the borehole wall (borehole $76 \mathrm{~mm}$ in diameter). In second model, the antenna is moved $20 \mathrm{~mm}$ and the outer surface is $10 \mathrm{~mm}$ from the borehole wall. In the third model, the antenna is moved $28 \mathrm{~mm}$ and the outer surface is $2 \mathrm{~mm}$ from the borehole wall. Figs. (17-19) present the effects of the movements.

The capacitive part of the impedance cycle (reactance is $<0.0 \Omega$ ) ends or the first resonances occurs at $\sim 1.1 \mathrm{MHz}$ at $\rho_{\mathrm{r}}=2500 \Omega \mathrm{m}$ and $\sim 1.6 \mathrm{MHz}$ at $\rho_{\mathrm{r}}=12500 \Omega \mathrm{m}$. This is followed by an inductive cycle (reactance is $>0 \Omega$ ). The resistances are $<200 \Omega$ in the middle frequencies of EMRE band and at the same time the scattering parameter $s_{11}$ has its highest values. Thus, the antenna would function effectively at the middle frequencies. At the favourable frequencies, $s_{11}$ values deviate less from each other $(<3 \mathrm{~dB})$ at all dipole positions, thus being insignificant (Fig. 17).

An increase in the rock relative permittivity value $\left(\varepsilon_{r r}=8\right.$ $\left.\rightarrow \varepsilon_{r r}=20\right)$ makes the functioning of the antenna more effective in the middle band, the highest scattering parameters being at the level of $-30.0 \mathrm{~dB}$ and meaning that a minimal part of the delivered power is reflected. The gap between the first resonances seems to be decreased and occur at 1.1 MHz. The effect of increasing the rock resistivity is to increase the resistances in the lower band $(<0.5 \mathrm{MHz})$, but the effect is not so remarked (Fig. 18).

A further increase in the rock relative permittivity value $\left(\varepsilon_{r r}=20 \rightarrow \varepsilon_{r r}=32\right.$ ) keeps the functioning of the antenna at the same level, the highest scattering parameters being at the level of $-25.0 \mathrm{~dB}$, meaning that a minimal part of the delivered power is reflected. The gap between the first resonance frequencies appears to be reduced and the resonances occur at $\sim 0.9 \mathrm{MHz}$. The resistances do not increase further in the lower band $(<0.5 \mathrm{MHz})$ when the rock resistivity increases (Fig. 19). The overall behaviour of the scattering parameter reveals that the insulated EMRE antenna of $40 \mathrm{~m}$ in length functions effectively in the middle frequencies of the EMRE band. The highest and lowest frequencies suffer the most from the borehole conditions. The differences in scattering parameter values in the favourable band have no relevance when compared with the losses generated by the internal attenuation of the rock medium.

The performance level of the system determines the maximum distance through the rock at which a signal can be detected. To estimate the maximum transmitter ranges in different situations, a simplified measurement geometry could be used $[14,30]$. In the normal situations where the host rock is highly resistive $(>10000 \Omega \mathrm{m})$, the borehole separation can be $\sim 1000$ metres and the signals are detectable at the lowest measurement frequency $(312.5 \mathrm{kHz})$, at least. However, at longer distances, the boreholes must 

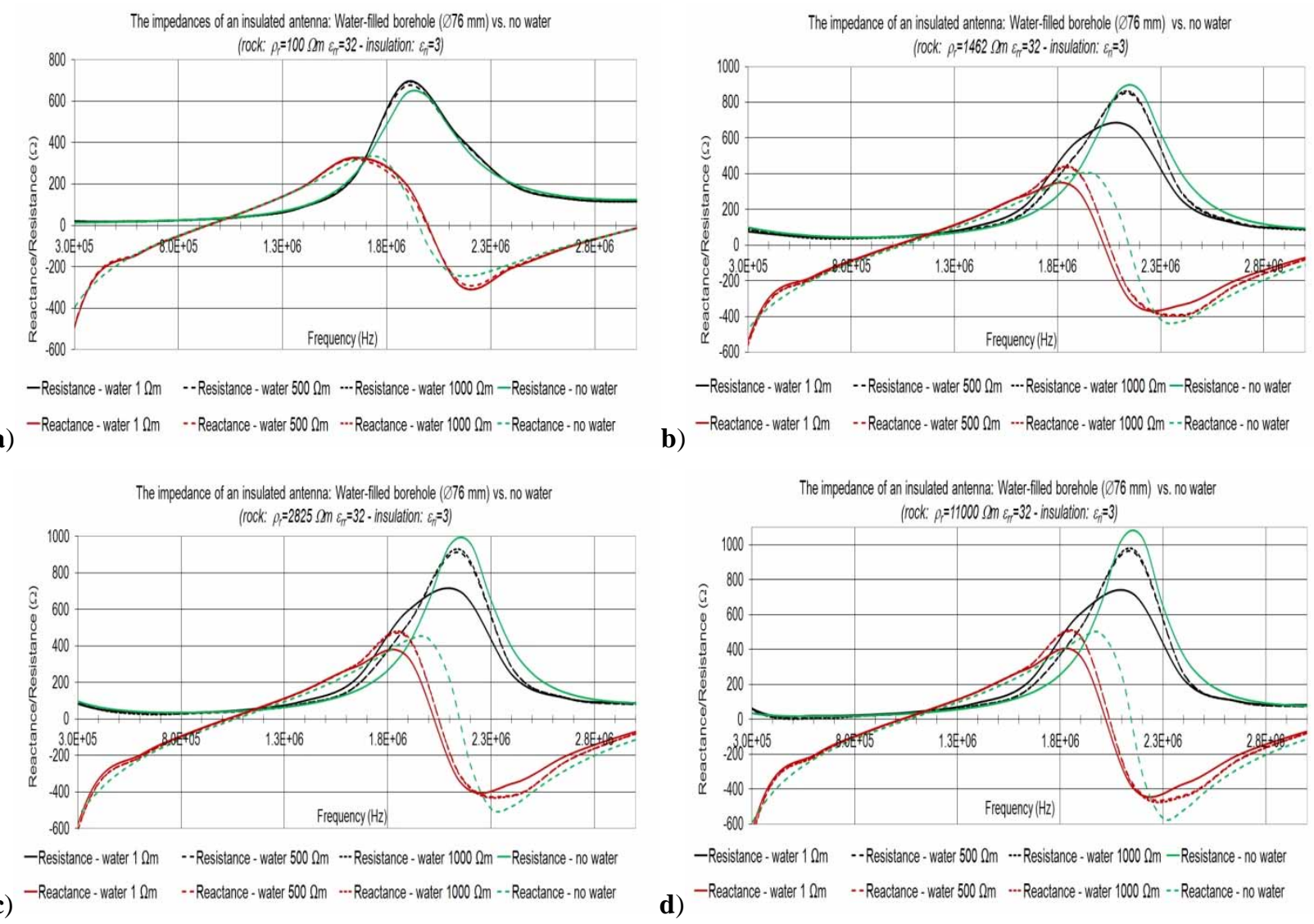

Fig. (15). Comparisons of a water-filled borehole and one without water. The input impedance of an antenna. Relative permittivities $\varepsilon_{\mathrm{rr}}=32$ for rock and $\varepsilon_{\mathrm{ri}}=3$ for insulation. Resistivities of the external medium: a) $\left.\left.\left.100 \Omega \mathrm{m}, \mathbf{b}\right) 1462 \Omega \mathrm{m}, \mathbf{c}\right) 2825 \Omega \mathrm{m}, \mathbf{d}\right) 11000 \Omega \mathrm{m}$. The solid lines represent resistances and the dashed lines reactances. The green lines represent the situation without a water layer.

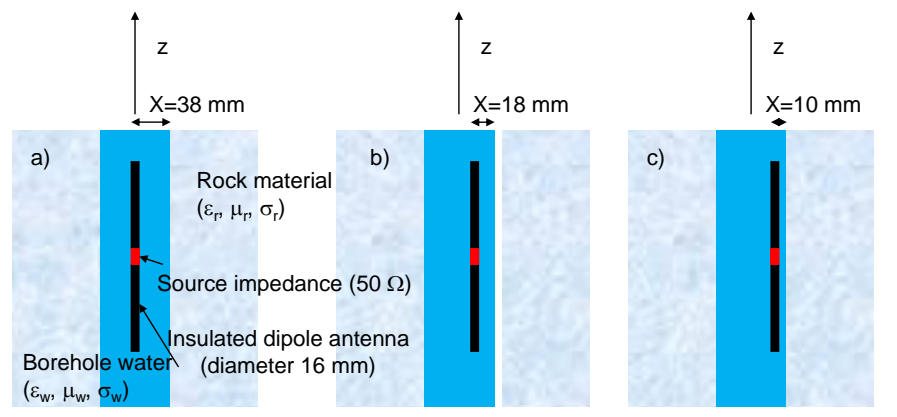

Fig. (16). An insulated antenna in a water-filled borehole. The diameter of the borehole is $76 \mathrm{~mm}$ and that of the antenna $16 \mathrm{~mm}$. The antenna is moved from its central position: on the left the outer surface is $30 \mathrm{~mm}$ from the borehole wall; in the middle the outer surface is 10 $\mathrm{mm}$ from borehole wall; on the right the outer surface is $2 \mathrm{~mm}$ from the borehole wall (not to scale).

also be deeper so that a reasonable angle coverage is met. It can said that when the transmitter and receiver are lowered deep enough, one can benefit from the depth gain, similarly to the situation where the dipoles are elevated above the ground surface, thereby benefitting from the height gain or allowing the antenna to operate more effectively (Fig. 3e). At borehole separations of 400-600 metres (host rock resistivity $\approx 7500 \Omega \mathrm{m}$ ), the EMRE system can be used across its whole frequency band, despite device-based losses, which had not been taken into account at all. It is the limited dynamic range of the device $(<40 \mathrm{~dB})$ that set limits for its use at short separation distances, when the saturation of the device can make usage impossible (Table 1).

\section{DISCUSSION AND CONCLUSIONS}

The operation of an antenna is strongly dependent on the medium in which the antenna exists. The main function of an antenna is to radiate and receive electromagnetic energy, serving as a transducer between the generator and the environment. The Q-factor determines the behaviour of the generated electromagnetic field in a medium, or whether the field propagates as waves or diffuses through the medium $[7,14,25]$. Fig. (4) presents the cut-off frequencies at which the diffusive movement turns into waves [14]. The most natural medium is air, in which modelling studies and the antenna characteristics for all antenna types are straightforward to calculate. However, even in air, when the 


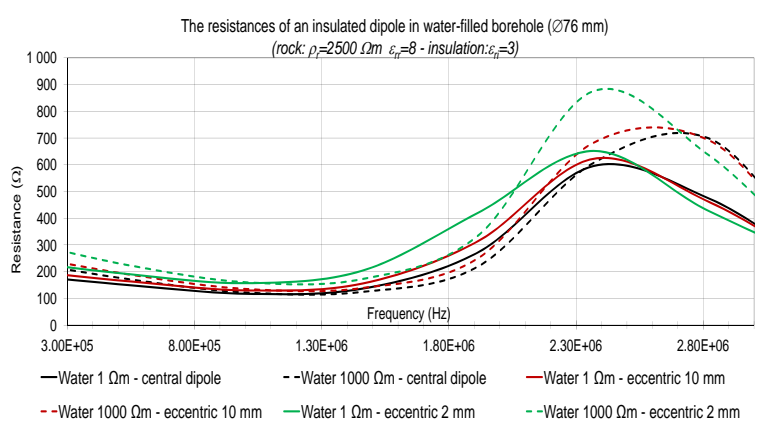

a)

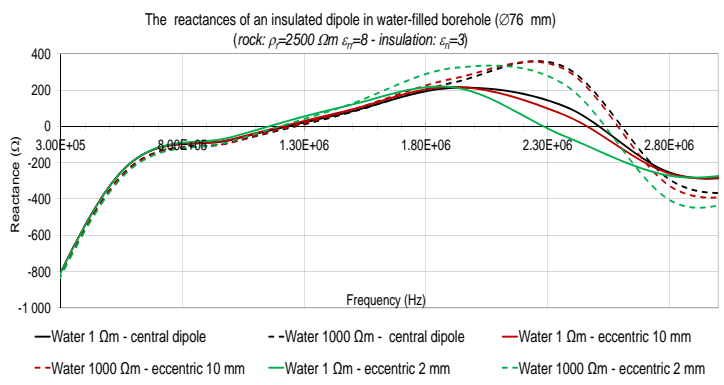

b)

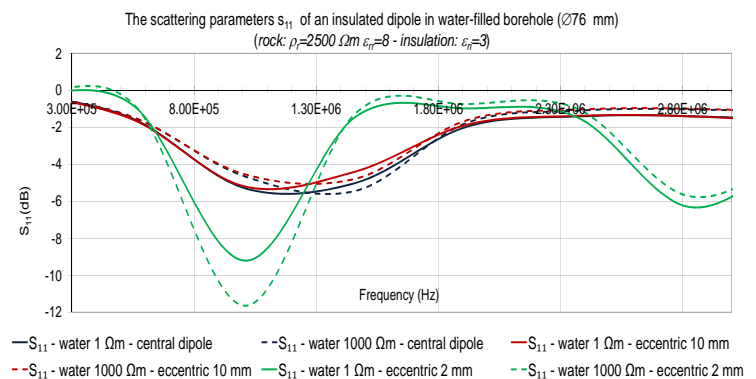

c)

Fig. (17). The components of antenna impedance $(R, X)$ and $s_{11}$ in a water-filled borehole $\left(\rho_{w}=1,1000 \Omega m\right)$. a) and d) Resistances $\left(\rho_{r}=\right.$ $2500 \Omega \mathrm{m}$ and $\left.\rho_{\mathrm{r}}=12500 \Omega \mathrm{m}\right)$. b) and e) Reactances $\left(\rho_{\mathrm{r}}=2500 \Omega \mathrm{m}\right.$ and $\left.\rho_{\mathrm{r}}=12500 \Omega \mathrm{m}\right)$. c) and f) Scattering parameters $\mathrm{s}_{11}\left(\rho_{\mathrm{r}}=2500 \Omega \mathrm{m}\right.$ and $\rho_{\mathrm{r}}=12500 \Omega \mathrm{m}$ ). Relative permittivities $\varepsilon_{\mathrm{rr}}=8$ for rock, $\varepsilon_{\mathrm{ri}}=3$ for antenna insulation, and $\varepsilon_{\mathrm{rw}}=81$ for borehole water.

antenna is placed near an interfering obstacle or the altitude from the Earth's surface is low, the operation may be severely disturbed (height gain). Power is lost as heat and the effectiveness is reduced when the antenna is near the surface $[21,24]$. Most of us have observed that the relocation of the device or a slight movement of the radio antenna makes the situation better in most cases.

When a bare metal wire antenna resides in a low resistive material, the current in the antenna will diminish along the antenna axis, because the surrounding material acts as a complementary path for the current. Thus, a metal wire could be used as a diagnostic probe to investigate the electric parameters of the borehole vicinity. In general, the antennas are highly insulated and the insulation will not only prevent the leakage of conducting charges, but also greatly reduces the sensitivity of the entire current distribution to the electrical properties of the ambient medium [22]. In Figs. (7,
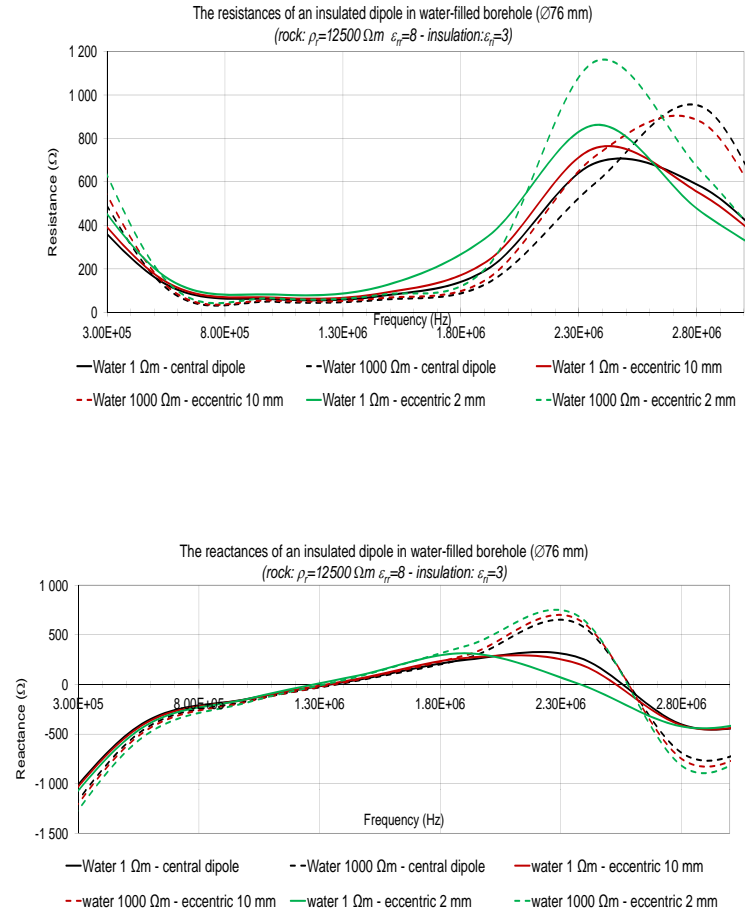

e)

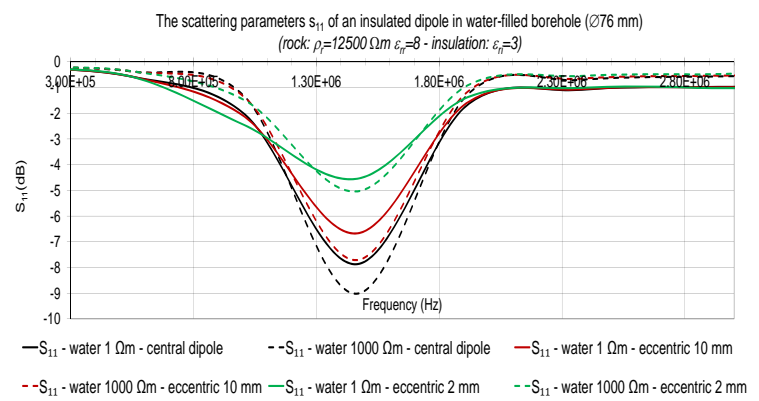

8), the current distribution of a metal wire and an insulated dipole antenna are compared as a function of the resistivity of borehole water and frequency. It is evident that the low resistivity of borehole water $(1 \Omega m)$ makes the surrounding material act as a complementary path for the current, and the current in a wire antenna will effectively diminish along the antenna axis. The effect of reducing the water resistivity $(1000 \Omega \mathrm{m}$ ) is to diminish the diagnostic power of the wire antenna. The thickness of the insulation layer effectively prevents the leakage of charges in the insulated antenna and the sensitivity to the electrical properties of the environment. In addition, the electrical length of an antenna is shorter than it should be in air. This is due to the difference in the electrical parameters: permittivity and resistivity. In air, the relative permittivity is $\varepsilon_{\text {rair }}=1$ and the resistivity $\sim \infty$, and the electromagnetic wave propagates at the speed of light. However, in a dissipative material, the parameters are 


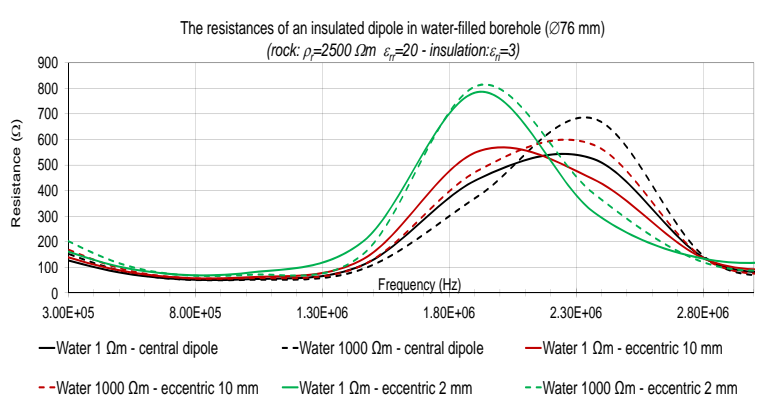

a)

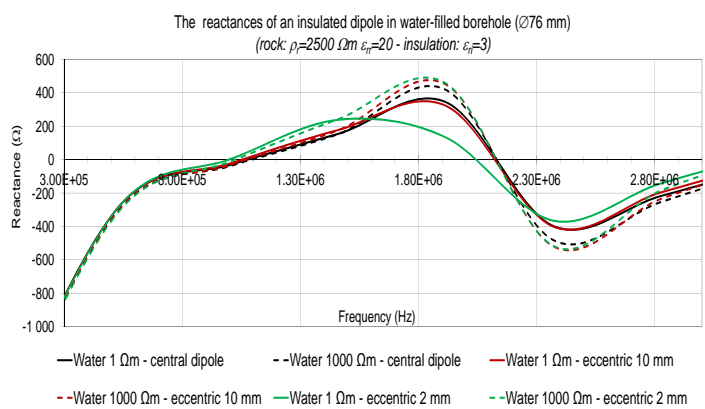

b)

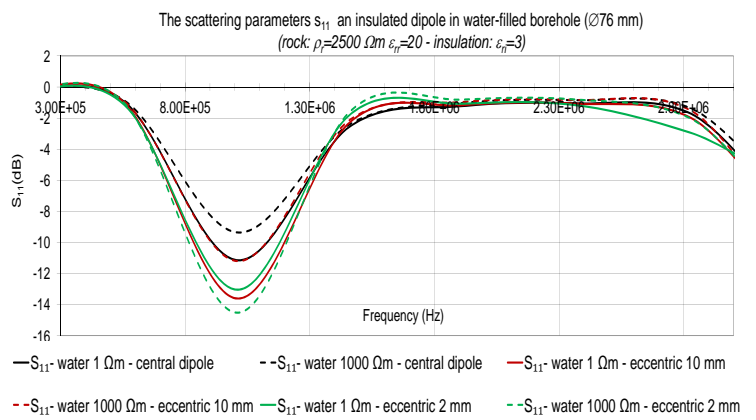

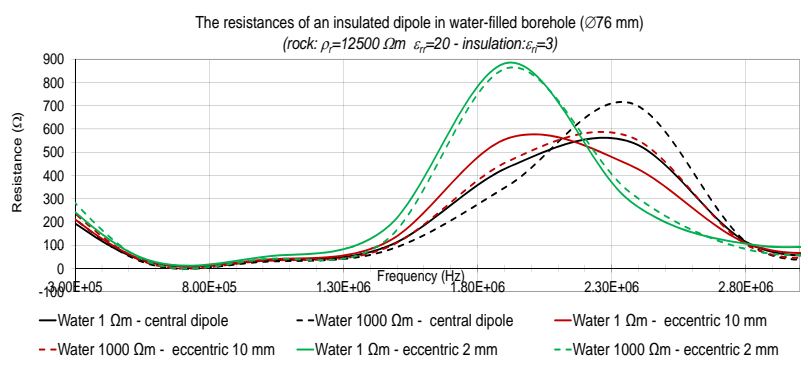

d)

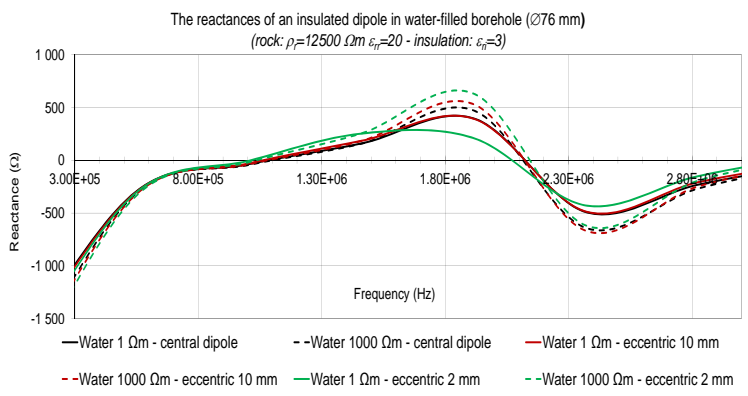

e)

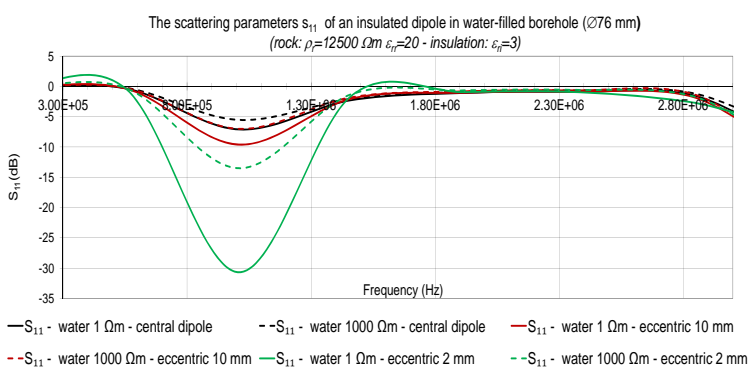

c)

f)

Fig. (18). The components of antenna impedance $(R, X)$ and $s_{11}$ in a water-filled borehole $\left(\rho_{w}=1,1000 \Omega m\right)$. a) and d) Resistances $\left(\rho_{r}=\right.$ $2500 \Omega \mathrm{m}$ and $\left.\rho_{\mathrm{r}}=12500 \Omega \mathrm{m}\right)$. b) and e) Reactances $\left(\rho_{\mathrm{r}}=2500 \Omega \mathrm{m}\right.$ and $\left.\rho_{\mathrm{r}}=12500 \Omega \mathrm{m}\right)$. c) and f) Scattering parameters $\mathrm{s}_{11}\left(\rho_{\mathrm{r}}=2500 \Omega \mathrm{m}\right.$ and $\rho_{\mathrm{r}}=12500 \Omega \mathrm{m}$ ). Relative permittivities $\varepsilon_{\mathrm{rr}}=20$ for rock, $\varepsilon_{\mathrm{ri}}=3$ for antenna insulation and $\varepsilon_{\mathrm{rw}}=81$ for borehole water.

different, or $\varepsilon_{\mathrm{rr}} \gg \varepsilon_{\mathrm{rair}}$, and the resistivity may range over several decades, varying from $10^{-8}$ to $10^{+14} \Omega \mathrm{m}$. Thus, the speed of the electromagnetic wave may be much lower and the wavelength is reduced by the $\mathrm{N}$-factor. The relative permittivity of the insulation layer is lower than in rock and the conductivity is slightly greater. However, the electrical length of the insulated antenna is shorter.

The mathematical treatment of the operation of an antenna when situated in a dissipative medium, such as in deep water-filled boreholes, becomes much worse. Modelling studies become complicated and numerical methods must be used. The traditional transmission line technique is feasible and the implementation is easy to perform, for instance in MatLab, but in deep water-filled boreholes, the models cannot include a water layer at all in WKG and CHEN models. Despite the lack of a water layer, the transmission line models can give valuable information on the operation of the antenna in a hostile environment. These models can be the first steps to becoming familiar with the behaviour of the antenna. On the other hand, the water layer is not the most important factor in the borehole models if an insulated antenna is considered in the EMRE band. The transmission line models (WKG, CHEN) were compared with the FEKO results in Figs. (11-13). There was a restriction on using the WKG/CHEN models, as a water layer could not be included in the models. An increase in the resistivity and relative permittivity of the rock has the effect on the resonance points, and the models approach each other. Due to the movement of the resonance points, the favourable resistance area $(<200 \Omega)$ becomes narrower in the EMRE band. When the resistivity of the rock is $\rho_{r}=11000 \Omega \mathrm{m}$ and the relative permittivity $\varepsilon_{\mathrm{rr}}=8$, the first resonance point occurs at $\sim 1.4 \mathrm{MHz}(W K G) / 1.6 \mathrm{MHz}(F E K O, C H E N)$. The resonance moves to a level of $<1 \mathrm{MHz}$ in all models when 


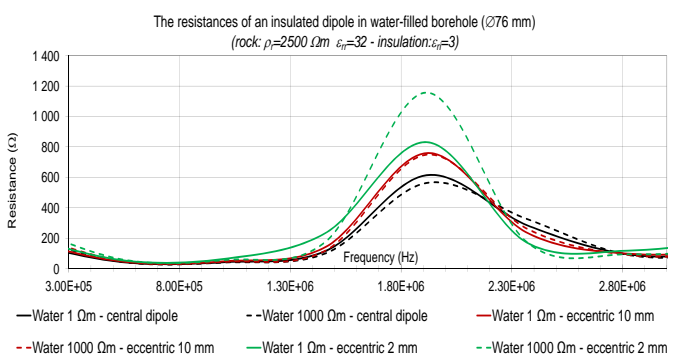

a)

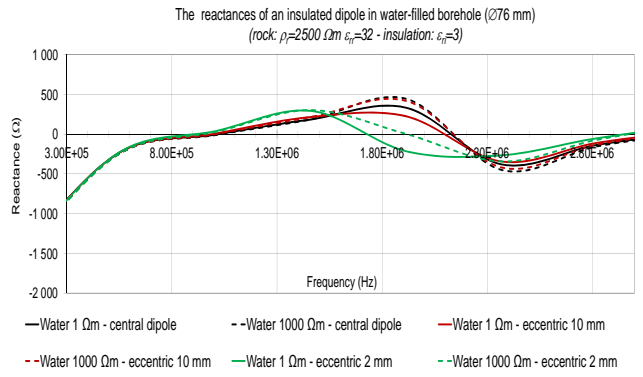

b)

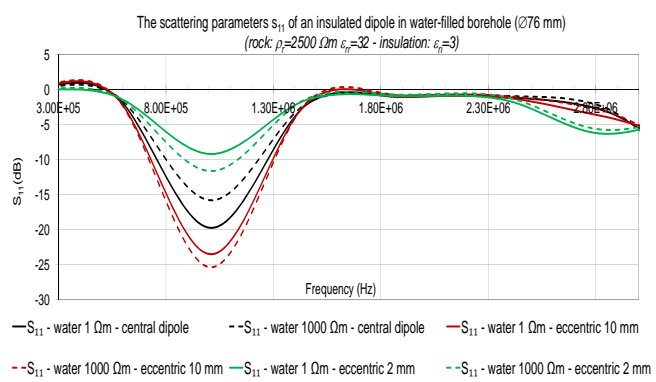

c)

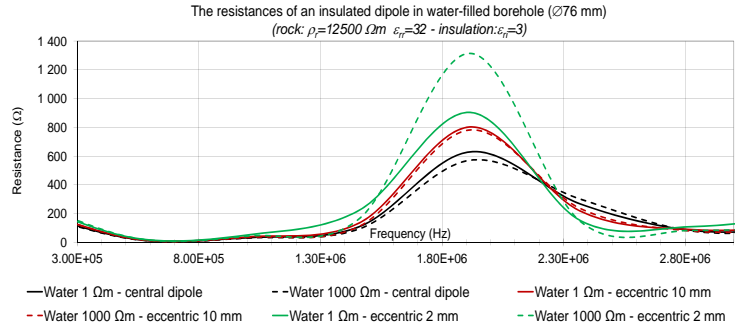

d)

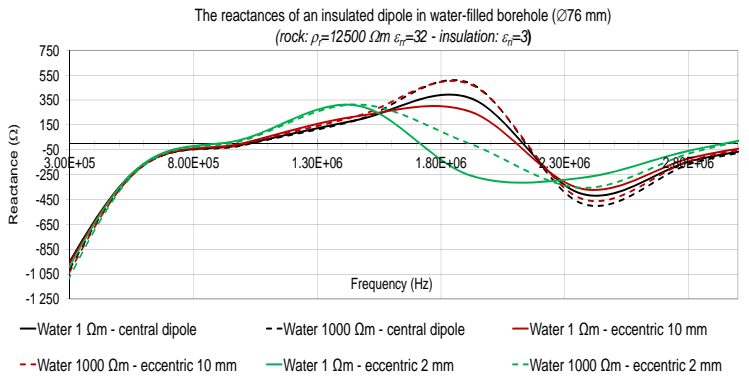

e)

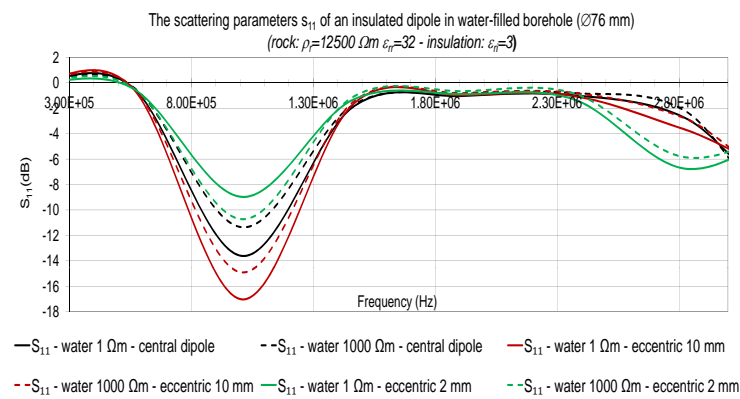

f)

Fig. (19). The components of antenna impedance $(R, X)$ and $s_{11}$ in a water-filled borehole $\left(\rho_{w}=1,1000 \Omega m\right)$. a) and d) Resistances $\left(\rho_{r}=\right.$ $2500 \Omega \mathrm{m}$ and $\left.\rho_{\mathrm{r}}=12500 \Omega \mathrm{m}\right)$. b) and e) Reactances $\left(\rho_{\mathrm{r}}=2500 \Omega \mathrm{m}\right.$ and $\left.\rho_{\mathrm{r}}=12500 \Omega \mathrm{m}\right)$. c) and f) Scattering parameters $\mathrm{s}_{11}\left(\rho_{\mathrm{r}}=2500 \Omega \mathrm{m}\right.$ and $\rho_{\mathrm{r}}=12500 \Omega \mathrm{m}$ ). Relative permittivities $\varepsilon_{\mathrm{rr}}=32$ for rock, $\varepsilon_{\mathrm{ri}}=3$ for antenna insulation and $\varepsilon_{\mathrm{rw}}=81$ for borehole water.

the rock's relative permittivity is $\varepsilon_{\mathrm{rr}}=32$. The second resonance point occurs at $\sim 3.0 \mathrm{MHz} / 3.8 \mathrm{MHz}\left(\rho_{r}=11000\right.$ $\left.\Omega m, \varepsilon_{r r}=8\right)$, moving to $1.8 \mathrm{MHz} / 2.1 \mathrm{MHz}\left(\rho_{r}=11000\right.$ $\left.\Omega m ; \varepsilon_{r r}=32\right)$. This means that between the first and second resonance point, the antenna operates inductively. The cycle continues and the capacitive period is followed. The behaviour of the transmission line models (WKG, CHEN) coincides quite well with the corresponding FEKO results.

More sophisticated tools must be used when more detailed models are designed (e.g. FEKO). The principle characteristics (e.g. power density, antenna pattern, radiation resistance) of an antenna can be calculated by using the same approach as in a non-conducting (nondissipative) medium, but the electrical parameters of the medium are now complex quantities and the results are different from one point to another (dependent on the radius $R$ ). The characteristic effect of the conducting medium on the antenna patterns is to broaden the pattern and swing the maximum direction of the pattern. Even the determination of the origin (central or end) may lead to confusing solutions in the conducting medium, and the modifications in antenna patterns become more pronounced due to a different viewpoint, unlike in air, where the coordinates of the origin in the proximity of an antenna yield the same result. The phase shift and the attenuation due to the contribution from one end of the antenna relative to that from the other end are not significant [21]. Boreholes are most typically waterfilled, and a water layer might influence the impedance and power delivery of an antenna. Because the water layer is thin, its influence is not perceptible in the EMRE band, and the most notable effects are generated by both the resistivity and permittivity of the rock and the permittivity of the insulation (Figs. 14, 15). Thus, when the resistivity and permittivity of the rock change, visible effects on the impedance of the antenna are evident and contribute to the power delivery, or more power may be lost as heat. 
Table 1. The Total Attenuation Losses (dB) as a Function of Material Parameters and Frequency. All Other Losses (e.g. in the Device, Different Polarizations in Transmission and Reception) are Excluded. Green Numbers are Referred to as Favourable Conditions.

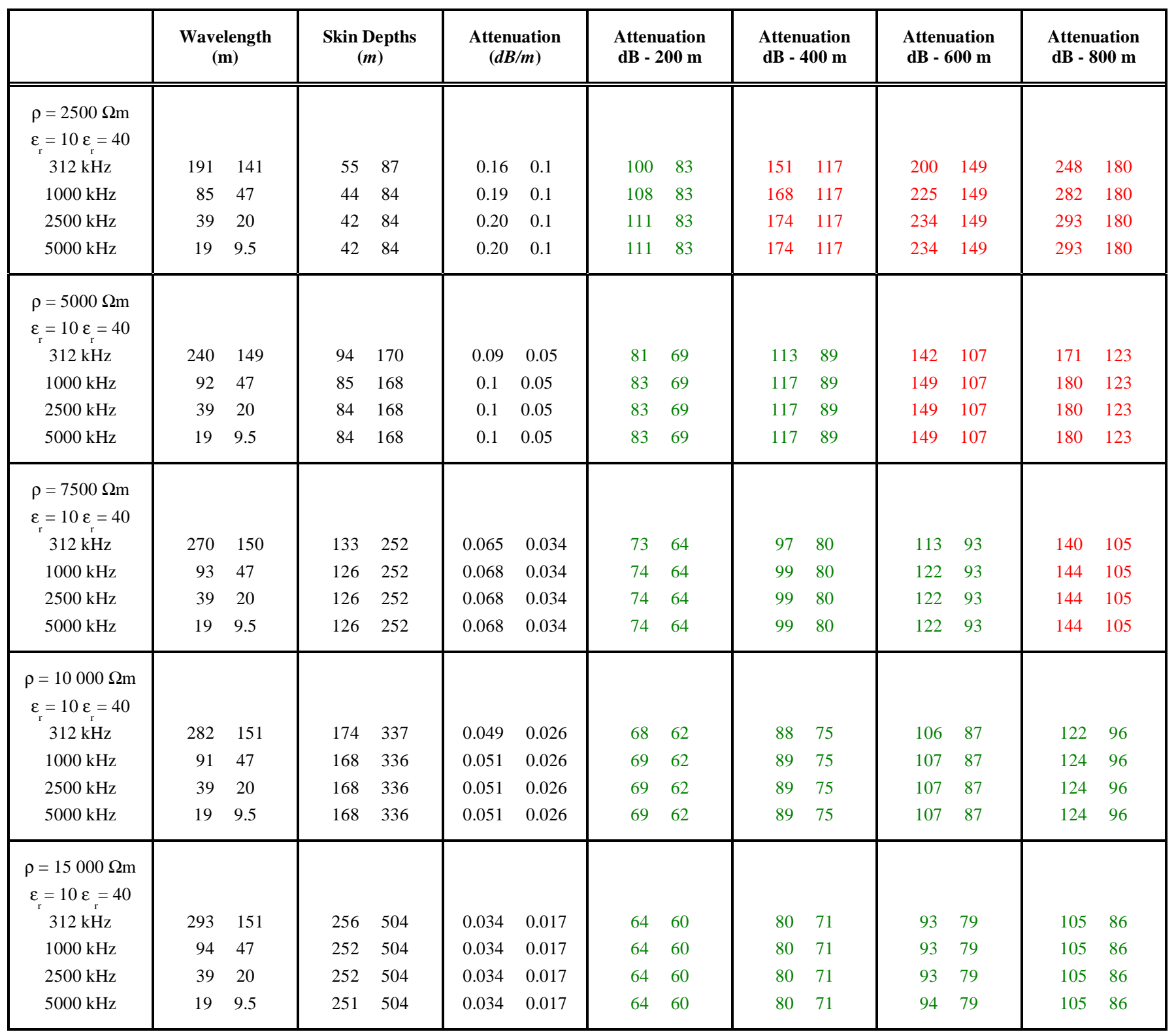

Vogt [2] has examined the operation of a borehole antenna in his dissertation, and the final conclusion was that the gain and directivity of an insulated antenna may be reasonable; thus, they are well suited for radio tomography applications. The disturbing effects of the air-earth interface can be avoided by starting the measurements (transmitter/receiver antenna) at least one dominant wavelength from the surface. Thus, when the transmitter and receiver are lowered deep enough, one would benefit from the depth gain, similar to the situation where the dipoles are elevated above the ground surface, benefitting from the height gain $[9,24]$. I have theoretically examined the variations in antenna impedance and scattering parameters $\mathrm{s}_{11}$. The highest frequency, $2500 \mathrm{kHz}$, suffers the most in the borehole conditions, but on the other hand, a halving of the transmitter power only means a decrease of $3 \mathrm{~dB}$. Thus, the borehole conditions alone are not the most important issue when considering the transmission of radio waves through geological transformations (Figs. 16-19) [14]. In the EMRE system, the transmitter is a continuous wave $(\mathrm{CW})$ device with a power of $2 \mathrm{~W}(33 \mathrm{dBm})$ and an estimated performance level of $\sim 135 \mathrm{~dB}$. According to the theoretical calculations, where a simplified geometry $[14,30]$ was used, it was possible to estimate the maximum transmitter ranges. The results imply that borehole separations could be up to 1000 $m$ when the lowest system frequency of $312.5 \mathrm{kHz}$ is used (Table 1) [16].

In summary, insulated EMRE antennas maintain their effectiveness and feasibility at an acceptable level in the typical borehole situations in the frequency band of 312.5$2500 \mathrm{kHz}$. On the contrary, the major factor that limits the use of radiofrequencies in geophysics is the strong absorption of energy in most earth materials, or the 
penetration may be highly frequency dependent. In addition, the first results from the field revealed that the EMRE system appears to operate according to theoretical expectations (Table 1), or even better.

\section{CONFLICT OF INTEREST}

The author confirms that this article content has no conflict of interest.

\section{ACKNOWLEDGEMENTS}

I wish to acknowledge RF specialist Mika Niemelä, who maintained the proper functioning of the EMRE device. Geophysicists Tapio Ruotoistenmäki and Kimmo Korhonen aided in the preparation of this paper.

\section{REFERENCES}

[1] Korpisalo A. Geotomographic studies for ore explorations with the EMRE system. (unpublished, part of a $\mathrm{PhD}$ thesis), 2012. [In Press].

[2] Vogt D. The modelling and design of Radio Tomography antennas. $\mathrm{PhD}$ thesis, unpublished, University of York (United Kingdom), 2000. Available http://www.opengrey.eu/item/display/10068/624 370

[3] Mahrer KD. Review of the radio frequency (RIM) method and its utilization in near-surface investigations. Leading Edge 1995; 249256.

[4] Buselli G. Electrical geophysics in the USSR. Geophysics 1980; 10: 1551-62.

[5] Redko GV, Lebedkin LV, Shuval-Sergeev A, Stevens K, Kazda G. Borehole electromagnetic geophysical methods in ore and coal deposits. A paper at the International Conference "300 years of Russian mine-geological service”, Saint-Petersburg (Russia), 2000. Available at: http://www.farasystem.ru/Pubs/Spb1998_Text_E.pdf.

[6] Stevens K, Watts A, Redko G. In-Mine Applications of the Radiowave Method in the Sudbury Igneous Complex, A paper at the International Conference "Mine Geophysics-98", SaintPetersburg (Russia), 1998. Available at: http://www.farasystem.ru/ Pubs/papr0398.pdf

[7] Jol HM. Ground penetrating radar: Theory and applications. 1st ed.; Elsevier science: Amsterdam: Netherlands 2009.

[8] Jackson MJ, Tweeton DR. 3DTOM-Three-dimensional geophysical tomography. USBM Report of Investigation 9617; 1996.

[9] deBettencourt JT, Surcliffe RA. Studies in deep strata radio communication. Raytheon Company. Final report 1962.

[10] Dokoupil S, Karpinsky J, Kaspar M. The attenuation of electromagnetic waves in rocks. Studia Geoph 1962; 6: 176-92.
[11] Stratton JA. Electromagnetic theory. McGraw-Hill Book Company: New York and London 1941.

[12] Zhang K, Li D. Electromagnetic Theory of Microwaves and Optoelectronics. 2nd ed., Springer-Verlag; Berlin Heidelberg, 2008.

[13] Lafleche PT. Underground UHF-EM Transillumination: A feasibility study. PhD studies. The department of Geological Sciences. McGill University, Montreal, Canada 1985.

[14] Korpisalo A. Characterization of geotomographic studies with the EMRE system. (unpublished, part of PhD thesis), 2012. [In Press].

[15] Korpisalo A, Jokinen T, Popov N, et al. Review of Crosshole Radiowave Imaging (FARA) in Borehole Sections OL-KR4-OLKR10 and OLKR10-OL-KR2 in Olkiluoto. Posiva Working report 2008-79, 2008

[16] Heikkinen E, Korpisalo A, Jokinen T, et al. Crosshole radiowave imaging (RIM) at Eurajoki Olkiluoto, Finland. A paper at the International Conference "Near Surface 2006 in Helsinki". EAGE, September 2006.

[17] Seybold JS. Introduction to RF propagation. John Wiley \& Sons Inc; New Jersey 2005.

[18] Ludwig R, Bretchko P. RF circuit design: Theory and application. Prentice Hall; Upper Saddle River 2009.

[19] FEKO. EM Software and Systems Ltd, FEKO user's manual, Suite 6.0, 2012.

[20] Collins Radio Company, Fundamentals of One Side Band. $2^{\text {nd }}$ Ed, 1 September, 1959. Available at: http://collinsradio.org/archives/ssb_ fundamentals/Fundamentals of Single Side Band-one-file.pdf

[21] Moore RK. Effects of a Surrounding Conducting Medium on Antenna Analysis. IEEE Trans Antennas Propagat 1963; 216-25.

[22] King RWP, Smith GS. Antennas in Materia: Fundamentals, Theory, and Applications. MIT Press, 1980.

[23] Holliger K, Musil M, Maurer HR. Ray-based amplitude tomography for cross-hole georadar: a numerical assessment. J App Geoph 2001; 47: 2856-298.

[24] Wait JR. Characteristics of antennas over lossy earth, in Antenna Theory, edited by Collin R.E. \& Zucker F.J. McGraw-Hill Book Company; New York: 1969; chap. 23-24, 386-514.

[25] Daniels DJ. Surface Penetrating Radar. The Institution of Electrical Engineers. London: 1996.

[26] Karlsson A. Physical limitations of antennas in a lossy medium. IEEE Trans Antennas Propag 2004; 52(8): 2027-33.

[27] Sato M, Thierbach R. Analysis of a Borehole Radar in Cross-hole Mode. IEEE Trans Geosci Remote Sens 1991; 29: 899-904.

[28] Gouws M. Modeling of a monostatic borehole radar antenna, McS thesis, University of Stellenbosch 2006.

[29] Gustrau F, Manteuffel D. EM Modeling of Antennas and RF Components for Wireless Communication Systems Berlin Heidelberg: Springer-Verlag 2006.

[30] Pralat A, Zdunek R. Electromagnetic Geotomography-Selection of Measuring Frequency. IEEE Sens J 2005; 5: 242-50.

(C) Arto Korpisalo Licensee Bentham Open.

This is an open access article licensed under the terms of the Creative Commons Attribution Non-Commercial License (http://creativecommons.org/licenses/by$\mathrm{nc} / 3.0 /$ ) which permits unrestricted, non-commercial use, distribution and reproduction in any medium, provided the work is properly cited. 